\title{
Homotopy Perturbation Method and Variational Iteration Method for Harmonic Waves Propagation in Nonlinear Magneto-Thermoelasticity with Rotation
}

\author{
Khaled A. Gepreel, ${ }^{1,2}$ S. M. Abo-Dahab, ${ }^{2,3}$ and T. A. Nofal ${ }^{2,4}$ \\ ${ }^{1}$ Math. Department, Faculty of Science, Zagazig University, Zagazig 44519, Egypt \\ ${ }^{2}$ Math. Department, Faculty of Science, Taif University, Saudi Arabia \\ ${ }^{3}$ Math. Department, Faculty of Science, SVU, Qena 83523, Egypt \\ ${ }^{4}$ Math. Department, Faculty of Science, El-Minia University, Egypt \\ Correspondence should be addressed to Khaled A. Gepreel, kagepreel@yahoo.com and \\ S. M. Abo-Dahab, sdahb@yahoo.com
}

Received 17 August 2011; Accepted 3 October 2011

Academic Editor: Cristian Toma

Copyright (C) 2012 Khaled A. Gepreel et al. This is an open access article distributed under the Creative Commons Attribution License, which permits unrestricted use, distribution, and reproduction in any medium, provided the original work is properly cited.

The homotopy perturbation method and variational iteration method are applied to obtain the approximate solution of the harmonic waves propagation in a nonlinear magneto-thermoelasticity under influence of rotation. The problem is solved in one-dimensional elastic half-space model subjected initially to a prescribed harmonic displacement and the temperature of the medium. The displacement and temperature are calculated for the methods with the variations of the magnetic field and the rotation. The results obtained are displayed graphically to show the influences of the new parameters and the difference between the methods' technique. It is obvious that the homotopy perturbation method is more effective and powerful than the variational iteration method.

\section{Introduction}

In the past recent years, much attentions have been devoted to simulate some real-life problems which can be described by nonlinear coupled differential equations using reliable and more efficient methods. The nonlinear coupled system of partial differential equations often appear in the study of circled fuel reactor, high-temperature hydrodynamics, and thermoelasticity problems, see [1-4]. From the analytical point of view, lots of work have been done for such systems. With the rapid development of nanotechnology, there appears an everincreasing interest of scientists and researchers in this field of science. Nanomaterials, because of their exceptional mechanical, physical, and chemical properties, have been the main topic 
of research in many scientific publications. Wave generation in nonlinear thermoelasticity problems has gained a considerable interest for its utilitarian aspects in understanding the nature of interaction between the elastic and thermal fields as well as for its applications. A lot of applications was paid on existence, uniqueness, and stability of the solution of the problem, see [5-7].

Much attention has been devoted to numerical methods, which do not require discretization of space-time variables or linearization of the nonlinear equations, among which the variational iteration method (VIM) suggested in [8-20] shows its remarkable merits over others. The method was successfully applied to a nonlinear one dimensional coupled equations in thermoelasticity [21], revealing that the method is very convenient, efficient, and accurate. The basic idea of variational iteration method is to construct a correction functional with a general Lagrange multiplier which can be identified optimally via variational theory.

The homotopy perturbation method $[8,22]$ has the merits of simplicity and easy execution. Unlike the traditional numerical methods, the HPM does not need discretization and linearization. Most perturbation methods assume that a small parameter exists, but most nonlinear problems have no small parameter at all. Many new methods have been proposed to eliminate the small parameter. Recently, the applications of homotopy theory among scientists appeared, and the homotopy theory becomes a powerful mathematical tool, when it is successfully coupled with perturbation theory. Sweilam and Khader [1] investigated variational iteration method for one dimensional nonlinear thermoelasticity. Applying He's variational iteration method for solving differential-difference equation is discussed by Yildirim [23]. Noor and Mohyud-Din [24], Mohyud-Din et al. [25-27] used He's polynomials or Padé approximants to solve solving higher-order nonlinear boundary value problems, second-order singular problems, and nonlinear boundary value problems. Mohyud-Din et al. [28] applied the modified variational iteration method for free-convective boundary-layer equation using Padé approximation. Mohyud-Din and Noor [29, 30] used Homotopy perturbation method for solving some new boundary value problems. MohyudDin et al. [31] investigated some relatively new techniques for nonlinear problems.

In this paper, the homotopy perturbation method and variational iteration method are used to solve the coupled harmonic waves nonlinear magneto-thermoelasticity equations under influence of rotation. The Maple and Mathematica software packages are used to obtain the approximate solutions in one-dimensional half-space. The displacement and temperature which obtained have been calculated numerically and presented graphically.

\section{Basic Idea of He's Homotopy Perturbation Method}

We illustrate the following nonlinear differential equation [8, 22]:

$$
A(u)-f(r)=0, \quad r \in \Lambda
$$

with the boundary conditions:

$$
B\left(u, \frac{\partial u}{\partial n}\right)=0, \quad r \in \Gamma,
$$


where $A$ is a general differential operator, $B$ is a boundary operator, $f(r)$ is an analytic function, and $\Gamma$ is the boundary of the domain $\Lambda$. Generally speaking, the operator $A$ can be divided into two parts which are $L$ and $N$, where $L$ is linear operator but $N$ is nonlinear operator. Equation (2.1) can therefore be rewritten as follows:

$$
L(u)+N(u)-f(r)=0 .
$$

By the homotopy technique, we construct a homotopy $V(r, p): \Lambda \times[0,1] \rightarrow R$ which satisfies

$$
H(V, p)=(1-p)\left[L(V)-L\left(u_{0}\right)\right]+p[A(V)-f(r)]=0, \quad r \in \Lambda,
$$

or

$$
H(V, p)=L(V)-L\left(u_{0}\right)+p L\left(u_{0}\right)+p(N(V)-f(r))=0, \quad r \in \Lambda,
$$

where $p \in[0,1]$ is an embedding parameter and $u_{0}$ is an initial approximation of (2.1) which satisfies the boundary conditions (2.2). Obviously, from (2.4) and (2.5) we have

$$
\begin{gathered}
H(V, 0)=L(V)-L\left(u_{0}\right)=0, \\
H(V, 1)=A(V)-f(r)=0 .
\end{gathered}
$$

The changing process of $p$ from zero to unity is just that of $V(r, p)$ from $u_{0}(r)$ to $u(r)$. In topology, this is called deformation, and $L(V)-L\left(u_{0}\right)$ and $A(V)-f(r)$ are called homotopy. According to the homotopy perturbation method, we can first use the embedding parameter " $p$ " as a small parameter and assume that the solution of (2.4) and (2.5) can be written as a power series in " $p$ " as follows:

$$
V=V_{0}+p V_{1}+p^{2} V_{2}+\cdots
$$

On setting $p=1$ results in the approximate solution of (2.3), we have

$$
u=\lim _{p \rightarrow 1} V=V_{0}+V_{1}+V_{2}+\cdots
$$

The combination of the perturbation method and the homotopy method is called the homotopy perturbation method, which has eliminated the limitations of the traditional perturbation methods. On the other hand, this technique can have full advantage of the traditional perturbation techniques. The series (2.8) is convergent to most cases. However, the convergent rate depends on the nonlinear operator $A(V)$.

(1) The second derivative of $N(V)$ with respect to $V$ must be small because the parameter may be relatively large, that is, $p \rightarrow 1$.

(2) The norm of $L^{-1}(\partial N / \partial V)$ must be smaller than one so that the series converges. 


\section{Application of Homotopy Perturbation Method on the Nonlinear Magneto-Thermoelastic with Rotation Equations}

In this section, we use the homotopy perturbation method to calculate the approximate solutions of the following nonlinear magneto-thermoelastic with rotation equations:

$$
\begin{gathered}
\left(1+\sigma_{1}\right) u_{t t}+\Omega u_{t}-u_{x x}\left(1-\sigma_{2}+2 \gamma u_{x}+3 \delta u_{x}^{2}\right)-\beta_{1} \theta_{x}-\beta_{2}\left(\theta u_{x}\right)_{x}=0 \\
\left(\theta-a u_{x}-\frac{1}{2} b u_{x}^{2}\right)_{t}-\left[\left(1+\alpha u_{x}\right) \theta_{x}\right]_{x}=0
\end{gathered}
$$

where $\gamma, \beta_{1}, \beta_{2}, a, b, \alpha$ are arbitrary constants, $\sigma_{1}, \sigma_{2}$ are the sensitive parts of the magnetic field, and $\Omega$ is the rotation parameter, with the initial conditions

$$
u(x, 0)=\theta(x, 0)=A(1-\cos (x)), \quad u_{t}(x, 0)=\theta_{t}(x, 0)=0,
$$

where $A$ is an arbitrary constant and the boundary conditions

$$
u(0, t)=\theta(0, t)=0, \quad u_{t}(0, t)=\theta_{t}(0, t)=0 .
$$

To investigate the traveling wave solution of (3.1), we first construct a homotopy perturbation method as follows:

$$
\begin{gathered}
(1-p)\left[\left(1+\sigma_{1}\right)\left(V_{t t}-V_{0 t t}\right)\right]+p\left[\left(1+\sigma_{1}\right) V_{t t}+\Omega V_{t}-V_{x x}\left(1-\sigma_{2}+2 \gamma V_{x}+3 \delta V_{x}^{2}\right)\right. \\
\left.-\beta_{1} \Theta_{x}-\beta_{2}\left(\Theta V_{x}\right)_{x}\right]=0, \\
(1-p)\left(\Theta_{t}-\Theta_{0 t}\right)+p\left[\Theta_{t}-a V_{x t}-b V_{x} V_{x t}-\Theta_{x x}-\alpha V_{x x} \Theta_{x}-\alpha V_{x} \Theta_{x x}\right]=0,
\end{gathered}
$$

where the initial approximations take the following form:

$$
\begin{aligned}
& V_{0}(x, t)=u_{0}(x, t)=u(x, 0)=A(1-\cos (x)), \\
& \Theta_{0}(x, t)=\theta_{0}(x, t)=\theta(x, 0)=A(1-\cos (x)) .
\end{aligned}
$$

According to the homotopy perturbation method, we can first use the embedding parameter " $p$ " as a small parameter and assume that the solution of (3.4) can be written as a power series in " $p$ " as the following:

$$
\begin{gathered}
V=V_{0}(x, t)+p V_{1}(x, t)+p^{2} V_{2}(x, t)+p^{3} V_{3}(x, t)+\cdots, \\
\Theta(x, t)=\Theta_{0}(x, t)+p \Theta_{1}(x, t)+p^{2} \Theta_{2}(x, t)+p^{3} \Theta_{3}(x, t)+\cdots,
\end{gathered}
$$

where $V_{j}$ and $\Theta_{j}, j=1,2,3, \ldots$ are functions to be determined. 
Substituting from (3.6) into (3.4) and arranging the coefficients of " $p$ " powers, we have

$$
\begin{aligned}
&\left(1+\sigma_{1}\right) V_{0, t t}+\left(\sigma_{1} V_{1, t t}-3 \delta V_{0, x}^{2} V_{0, x x}-\beta_{2} V_{0, x x} \Theta_{0}-2 \gamma V_{0, x} V_{0, x x}+\sigma_{2} V_{0, x x}-\beta_{1} \Theta_{0, x}\right. \\
&\left.-V_{0, x x}-\beta_{2} V_{0, x} \Theta_{0, x}+\Omega V_{0, t}+V_{1, t t}\right) p \\
&+\left(\sigma_{1} V_{2, t t}-2 \gamma V_{1, x} V_{0, x x}+V_{2, t t}-2 \gamma V_{0, x} V_{1, x x}-6 \delta V_{0, x} V_{1, x} V_{0, x x}-V_{1, x x}-\beta_{2} V_{1, x x} \Theta_{0}\right. \\
&\left.+\beta_{1} \Theta_{1, x}-\beta_{2} V_{0, x} \Theta_{1, x}-\beta_{2} V_{1, x} \Theta_{0, x}-\beta_{2} V_{0, x x} \Theta_{1}+\Omega V_{1, t}-3 \delta V_{0, x}^{2} V_{1, x x}+\sigma_{2} V_{1, x x}\right) p^{2} \\
&+\left(V_{3, t t}-\beta_{2} V_{2, x} \Theta_{0, x}-\beta_{2} V_{2, x x} \Theta_{0}-2 \gamma V_{2, x} V_{0, x x}-2 \gamma V_{1, x} V_{1, x x}+\sigma_{1} V_{3, t t}-\beta_{1} \Theta_{2, x}\right. \\
&+-6 \delta V_{0, x} V_{2, x} V_{0, x x}-3 \delta V_{1, x}^{2} V_{0, x x}+\sigma_{2} V_{2, x x}+\Omega V_{2, t}-V_{2, x x}-3 \delta V_{0, x}^{2} V_{2, x x}-2 \gamma V_{0, x} V_{2, x x} \\
&\left.-\beta_{2} V_{1, x x} \Theta_{1}-\beta_{2} V_{0, x x} \Theta_{2}-\beta_{2} V_{0, x} \Theta_{2, x}-6 \delta V_{0, x} V_{1, x} V_{1, x x}-\beta_{2} V_{1, x} \Theta_{1, x}\right) p^{3}+\cdots=0, \\
& \Theta_{0, t}+\left(-a V_{0, x t}-\alpha V_{0, x} \Theta_{0, x x}+\Theta_{1, t}-\alpha V_{0, x x} \Theta_{0, x}-2 b V_{0, x} V_{0, x t}-\Theta_{0, x x}\right) p \\
&+\left(\Theta_{2, t}-\Theta_{1, x x}-a V_{1, x t}-\alpha V_{1, x x} \Theta_{0, x}-2 b V_{1, x} V_{0, x t}-\alpha V_{0, x} \Theta_{1, x x}-\alpha V_{1, x} \Theta_{0, x x}\right. \\
&\left.-2 b V_{0, x} V_{1, x t}-\alpha V_{0, x x} \Theta_{1, x}\right) p^{2} \\
& \times\left(-\alpha V_{1, x x} \Theta_{1, x}-\alpha V_{2, x x} \Theta_{0, x}-2 b V_{2, x} V_{0, x t}-\alpha V_{0, x x} \Theta_{2, x}-2 b V_{1, x} V_{1, x t}-a V_{2, x t}\right. \\
&+\left.+\Theta_{3, t}-2 b V_{0, x} V_{2, x t}-\alpha V_{0, x} \Theta_{2, x x}-\alpha V_{1, x} \Theta_{1, x x}-\alpha V_{2, x} \Theta_{0, x x}-\Theta_{2, x x}\right) p^{3}+\cdots=0 .
\end{aligned}
$$

In order to obtain the unknowns of $V_{j}$ and $\Theta_{j},(j=1,2,3, \ldots)$, we construct and solve the following system considering the initial conditions (3.2):

$$
\begin{aligned}
& \sigma_{1} V_{1, t t}-3 \delta V_{0, x}^{2} V_{0, x x}-\beta_{2} V_{0, x x} \Theta_{0}-2 \gamma V_{0, x} V_{0, x x}+\sigma_{2} V_{0, x x}-\beta_{1} \Theta_{0, x}-V_{0, x x} \\
&-\beta_{2} V_{0, x} \Theta_{0, x}+\Omega V_{0, t}+V_{1, t t}=0 \\
& \sigma_{1} V_{2, t t}-2 \gamma V_{1, x} V_{0, x x}+V_{2, t t}-2 \gamma V_{0, x} V_{1, x x}-6 \delta V_{0, x} V_{1, x} V_{0, x x}-V_{1, x x}-\beta_{2} V_{1, x x} \Theta_{0} \\
&-\beta_{1} \Theta_{1, x}-\beta_{2} V_{0, x} \Theta_{1, x}-\beta_{2} V_{1, x} \Theta_{0, x}-\beta_{2} V_{0, x x} \Theta_{1}+\Omega V_{1, t}-3 \delta V_{0, x}^{2} V_{1, x x}+\sigma_{2} V_{1, x x}=0, \\
& V_{3, t t}- \beta_{2} V_{2, x} \Theta_{0, x}-\beta_{2} V_{2, x x} \Theta_{0}-2 \gamma V_{2, x} V_{0, x x}-2 \gamma V_{1, x} V_{1, x x}+\sigma_{1} V_{3, t t}-\beta_{1} \Theta_{2, x} \\
&- 6 \delta V_{0, x} V_{2, x} V_{0, x x}-3 \delta V_{1, x}^{2} V_{0, x x}+\sigma_{2} V_{2, x x}+\Omega V_{2, t}-V_{2, x x}-3 \delta V_{0, x}^{2} V_{2, x x}-2 \gamma V_{0, x} V_{2, x x} \\
&- \beta_{2} V_{1, x x} \Theta_{1}-\beta_{2} V_{0, x x} \Theta_{2}-\beta_{2} V_{0, x} \Theta_{2, x}-6 \delta V_{0, x} V_{1, x} V_{1, x x}-\beta_{2} V_{1, x} \Theta_{1, x}=0, \\
&-a V_{0, x t}-\alpha V_{0, x} \Theta_{0, x x}+\Theta_{1, t}-\alpha V_{0, x x} \Theta_{0, x}-2 b V_{0, x} V_{0, x t}-\Theta_{0, x x}=0, \\
& \Theta_{2, t}-\Theta_{1, x x}-a V_{1, x t}-\alpha V_{1, x x} \Theta_{0, x}-2 b V_{1, x} V_{0, x t}-\alpha V_{0, x} \Theta_{1, x x}-\alpha V_{1, x} \Theta_{0, x x} \\
&-2 b V_{0, x} V_{1, x t}-\alpha V_{0, x x} \Theta_{1, x}=0, \\
&-\alpha V_{1, x x} \Theta_{1, x}-\alpha V_{2, x x} \Theta_{0, x}-2 b V_{2, x} V_{0, x t}-\alpha V_{0, x x} \Theta_{2, x}-2 b V_{1, x} V_{1, x t}-a V_{2, x t}+\Theta_{3, t} \\
& \quad-2 b V_{0, x} V_{2, x t}-\alpha V_{0, x} \Theta_{2, x x}-\alpha V_{1, x} \Theta_{1, x x}-\alpha V_{2, x} \Theta_{0, x x}-\Theta_{2, x x}=0 .
\end{aligned}
$$


Consequently, we deduce after some calculations the following results:

$$
\begin{aligned}
& u=\lim _{p \rightarrow 1} V=V_{0}+V_{1}+V_{2}+\cdots, \\
& \theta=\lim _{p \rightarrow 1} \Theta=\Theta_{0}+\Theta_{1}+\Theta_{2}+\cdots,
\end{aligned}
$$

where

$$
\begin{aligned}
& V_{0}=A(1-\cos x), \\
& V_{1}=\frac{t^{2}}{2\left(4+4 \sigma_{1}\right)}\left[4 \gamma A^{2} \sin 2 x+3 \delta A^{3} \cos x-3 \delta A^{3} \cos 3 x+4 \beta_{2} A^{2} \cos x\right. \\
& \left.-4 \beta_{2} A^{2} \cos 2 x 4 \sigma_{2} A \cos x+4 \beta_{1} A \sin x+4 A \cos x\right], \\
& V_{2}=\frac{t^{4}}{32\left(\sigma_{1}+1\right)^{2}}\left\{\left(-\frac{8}{3} \beta_{2}^{2} A^{3}-\frac{8}{3} \beta_{2} A^{2}-\frac{8}{3} \gamma^{2} A^{3}-4 \delta A^{3}+4 A^{3} \sigma_{2} \delta+\frac{8}{3} \sigma_{2} A-\frac{9}{2} \delta^{2} A^{5}-\frac{4}{3} A \sigma_{2}^{2}\right.\right. \\
& \left.-\frac{4}{3} A+\frac{8}{3} A^{2} \sigma_{2} \beta_{2}-4 \beta_{2} A^{4} \delta\right) \cos x \\
& -\left(\frac{4}{3} \beta_{1} A+\delta A^{3} \beta_{1}+\frac{4}{3} \gamma A^{3} \beta_{2}-\frac{4}{3} A \sigma_{2} \beta_{1}+\frac{4}{3} \beta_{2} A^{2} \beta_{1}\right) \sin x \\
& +\left(-8 \gamma A^{2}+8 A^{2} \sigma_{2} \gamma-8 \beta_{2} A^{3} \gamma+\frac{4}{3} \beta_{2} A^{2} \beta_{1}-16 \delta A^{4} \gamma\right) \sin 2 x \\
& +\left(12 \gamma A^{3} \beta_{2}+3 \delta A^{3} \beta_{1}\right) \sin 3 x+20 \delta A^{4} \gamma \sin 4 x \\
& +\left(-\frac{20}{3} A^{2} \sigma_{2} \beta_{2}+\frac{20}{3} \beta_{2}^{2} A^{3}+\frac{8}{3} \gamma A^{2} \beta_{1}+\frac{20}{3} \beta_{2} A^{2}+6 \delta A^{4} \beta_{2}\right) \cos 2 x \\
& +\left(\frac{63}{4} \delta^{2} A^{5}-4 \beta_{2}^{2} A^{3}+12 \delta A^{3}+12 \beta_{2} A^{4} \delta+8 \gamma^{2} A^{3}-12 A^{3} \sigma_{2} \delta\right) \cos 3 x \\
& \left.-14 \beta_{2} A^{4} \delta \cos 4 x-\frac{45}{4} \delta^{2} A^{5} \cos 5 x\right\} \\
& +\frac{t^{3}}{32\left(\sigma_{1}+1\right)^{2}}\left\{\left(-\frac{16}{3} \Omega A \beta_{1}-\frac{16}{3} \beta_{1} A \sigma_{1}-\frac{8}{3} \beta_{2} \alpha \sigma_{1} A^{3}-\frac{16}{3} \beta_{1} A-\frac{8}{3} \beta_{2} A^{3} \alpha\right) \sin x\right. \\
& -\frac{16}{3} \Omega A^{2} \gamma \sin 2 x+\left(8 \beta_{2} A^{3} \alpha \sigma_{1}+8 \beta_{2} A^{3} \alpha\right) \sin 3 x \\
& +\left(\frac{16}{3} \Omega A \sigma_{2}-4 \Omega A^{3} \delta-\frac{16}{3} \Omega A^{2} \beta_{2}-\frac{16}{3} \Omega A\right) \cos (x) \\
& +\left(\frac{16}{3} \beta_{2} A^{2}+\frac{32}{3} \beta_{1} A^{2} \alpha \sigma_{1}+\frac{16}{3} \Omega A^{2} \beta_{2}+\frac{16}{3} \beta_{2} A^{2} \sigma_{1}+\frac{32}{3} \beta_{1} A^{2} \alpha\right) \cos 2 x \\
& \left.+4 \Omega A^{3} \delta \cos 3 x\right\},
\end{aligned}
$$




$$
\begin{aligned}
& \Theta_{0}=A(1-\cos (x)), \\
& \Theta_{1}=A^{2} t \alpha \sin (2 x)+A \cos (x) t, \\
& \Theta_{2}=\frac{A}{2\left(1+\sigma_{1}\right)}\left(\frac { t ^ { 3 } } { 3 } \left(12 \alpha A^{2} \gamma \cos ^{3}(x)+2 \alpha A \cos ^{2}(x) \beta_{1}+36 \alpha A^{3} \delta \sin (x) \cos ^{3}(x)\right.\right. \\
& +12 \alpha A^{2} \beta_{2} \sin (x) \cos ^{2}(x)+2 \alpha A \sigma_{2} \sin (x) \cos (x)-24 \alpha A^{3} \delta \sin (x) \cos (x) \\
& -2 \alpha A^{2} \beta_{2} \sin (x) \cos (x)-2 \alpha A \sin (x) \cos (x)-\alpha A \beta_{1}-10 \alpha \gamma A^{2} \cos (x) \\
& \left.-4 \alpha \beta_{2} A^{2} \sin (x)\right) \\
& +\frac{t^{2}}{2}\left(-2 \cos (x)+18 a \delta A^{2} \sin (x) \cos ^{2}(x)+16 b A^{2} \gamma \sin (x) \cos ^{2}(x)\right. \\
& +8 a \beta_{2} A \sin (x) \cos (x)+2 a \sigma_{2} \sin (x)+2 a \beta_{1} \cos (x)+24 \alpha^{2} A^{2} \sigma_{1} \cos ^{3}(x) \\
& -36 b A^{3} \delta \cos ^{4}(x)-16 b A^{2} \beta_{2} \cos ^{3}(x)+48 b A^{3} \delta \cos ^{2}(x)+4 b A^{2} \beta_{2} \cos ^{2}(x) \\
& +8 \cos ^{2}(x) a \gamma A-4 \cos (x)^{2} b A \sigma_{2}-20 \alpha^{2} A^{2} \cos (x)+24 \cos (x)^{3} \alpha^{2} A^{2} \\
& +4 b A \cos ^{2}(x)-4 b A-6 a \delta A^{2} \sin (x)-20 \alpha A \sin (x) \cos (x) \\
& -2 a \beta_{2} A \sin (x)-4 b A^{2} \beta_{2}-12 b A^{3} \delta+4 b A \sigma_{2}-8 b A^{2} \sin (x) \gamma \\
& +16 b A^{2} \beta_{2} \cos (x)-20 \alpha^{2} A^{2} \cos (x) \sigma_{1}-4 a \gamma A-2 \cos (x) \sigma_{1} \\
& \left.\left.-2 a \sin (x)+4 b A \beta_{1} \sin (x) \cos (x)-20 \alpha A \sin (x) \cos (x) \sigma_{1}\right)\right) .
\end{aligned}
$$

Now we make calculations for the results obtained by the homotopy perturbation method using the Maple software package with the following arbitrary constants:

$$
a=0.5, \quad A=0.001, \quad b=0.5, \quad \alpha=1, \quad \beta_{1}=\beta_{2}=0.05, \quad \gamma=1, \quad \delta=0.8
$$

The results obtained in (3.9) are displayed graphically in Figures 1-4.

\subsection{Special Cases}

(1) If we take into our consideration the first iteration (i.e., $u=V_{0}+V_{1}$ and $\theta=\Theta_{0}+\Theta_{1}$ ). See Figures 5, 6, 7, and 8.

(2) If the magnetic field and rotation are neglected, the components of the displacement $u$ and temperature $\theta$ take the following forms. See Figures 9 and 10. 


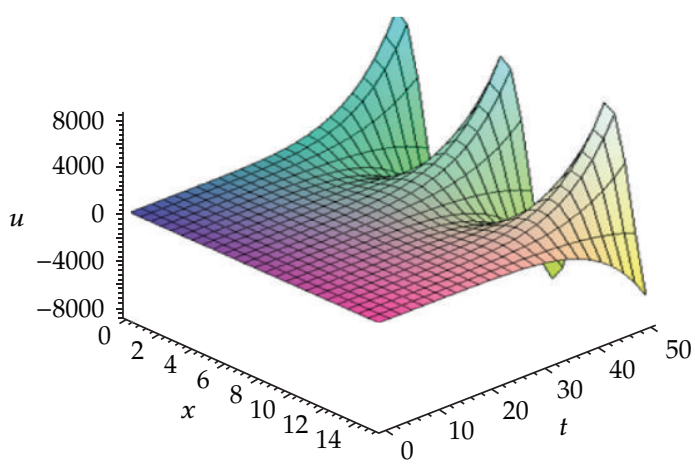

(a)

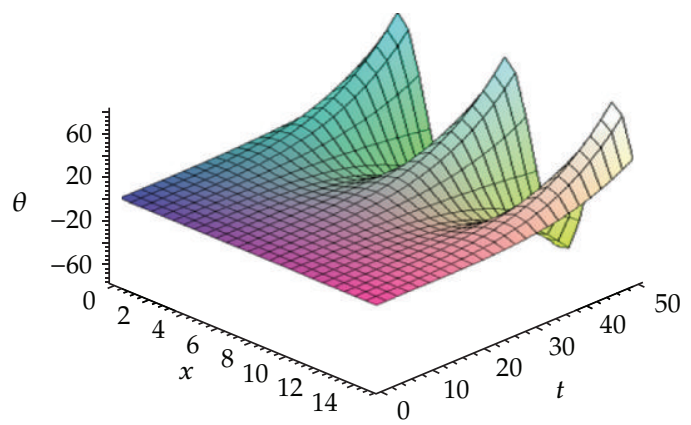

(b)

Figure 1: Variations of the displacement $u$ and temperature $\theta$ for various values of the axis $x$ and time $t$ when $\Omega=0.1, \sigma_{1}=0.2, \sigma_{2}=0.1$.

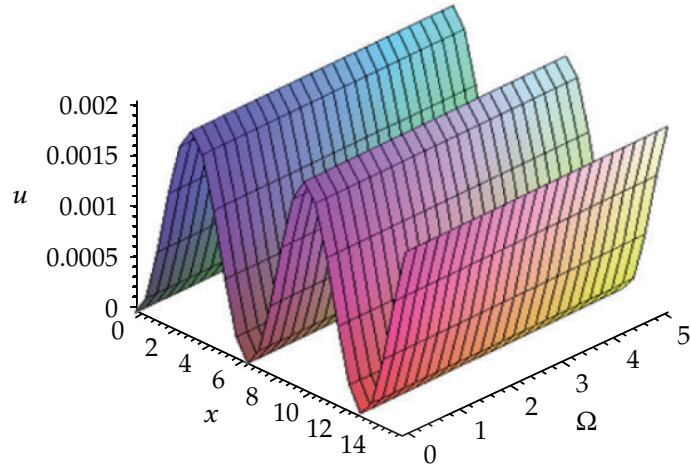

(a)

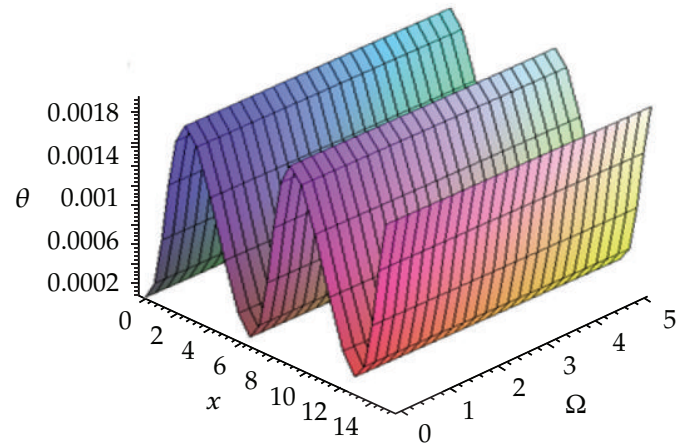

(b)

Figure 2: Variations of the displacement $u$ and temperature $\theta$ for various values of the axis $x$ and rotation $\Omega$ when $t=0.1, \sigma_{1}=0.2, \sigma_{2}=0.1$.

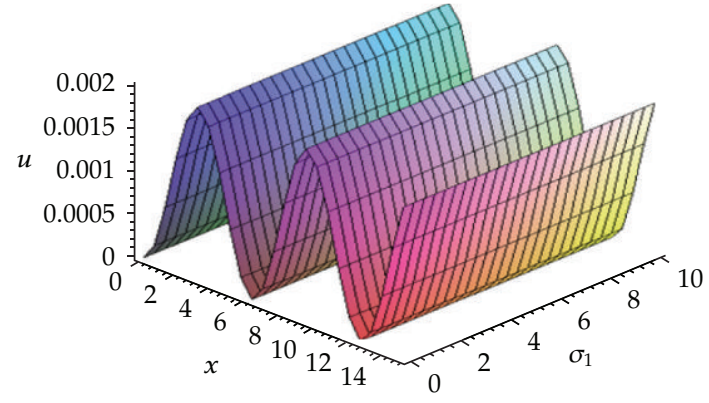

(a)

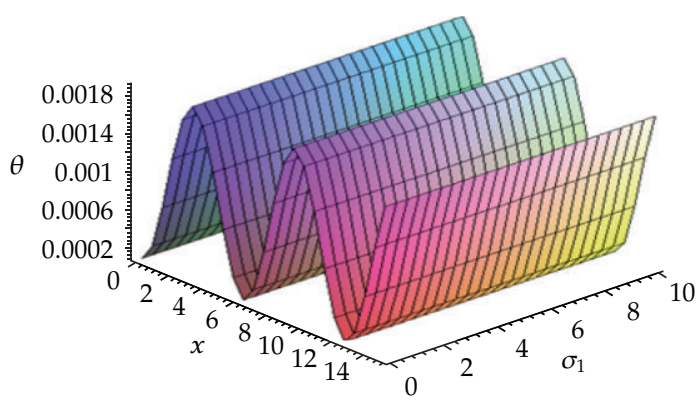

(b)

Figure 3: Variations of the displacement $u$ and temperature $\theta$ for various values of the axis $x$ and magnetic field $\sigma_{1}$ when $t=0.1, \Omega=0.1, \sigma_{2}=0.1$. 


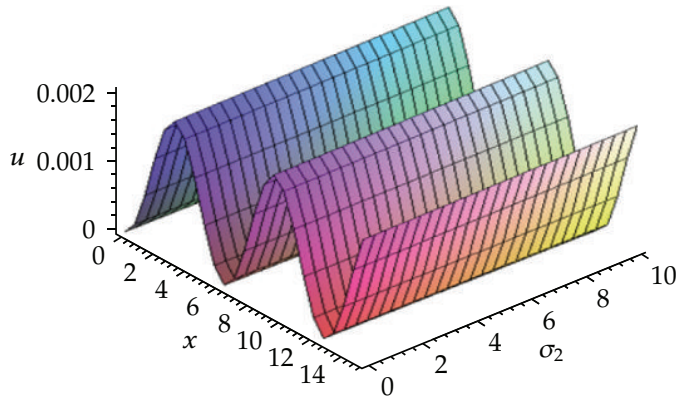

(a)

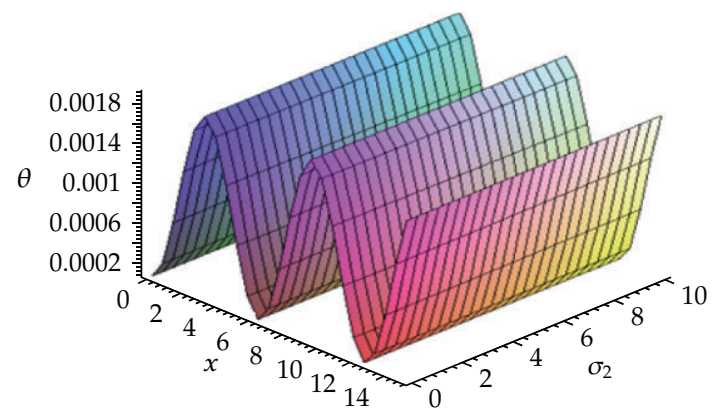

(b)

Figure 4: Variations of the displacement $u$ and temperature $\theta$ for various values of the axis $x$ and magnetic field $\sigma_{2}$ when $t=0.1, \Omega=0.1, \sigma_{1}=0.1$.

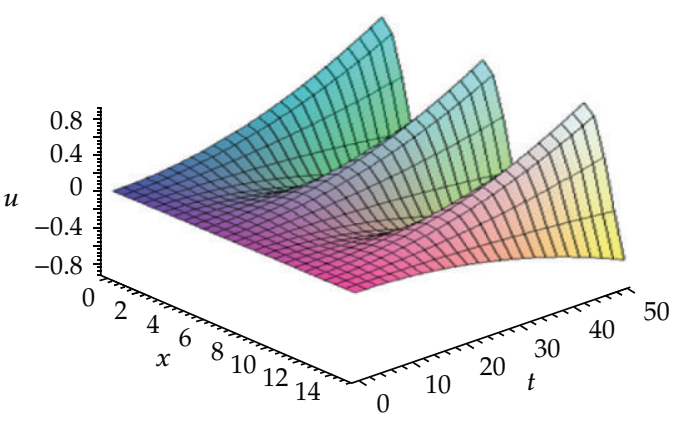

(a)

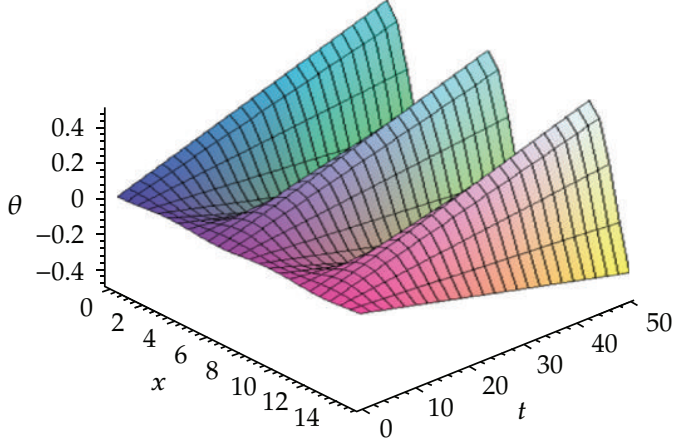

(b)

Figure 5: Variations of the displacement $u$ and temperature $\theta$ for various values of the axis $x$ and time $t$ when $\Omega=0.1, \sigma_{1}=0.2, \sigma_{2}=0.1$.

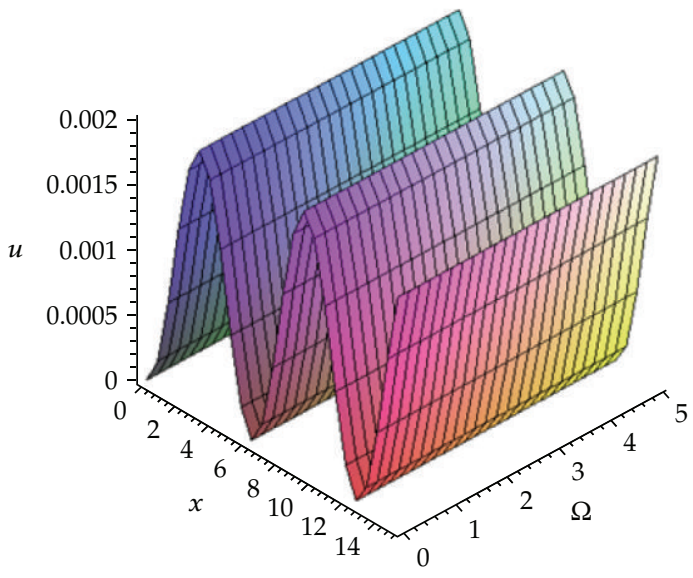

(a)

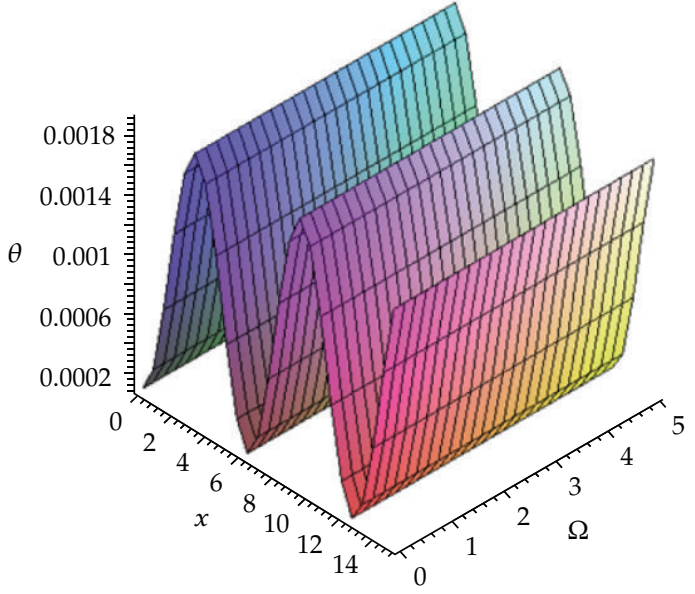

(b)

Figure 6: Variations of the displacement $u$ and temperature $\theta$ for various values of the axis $x$ and rotation $\Omega$ when $t=0.1, \sigma_{1}=0.2, \sigma_{2}=0.1$. 


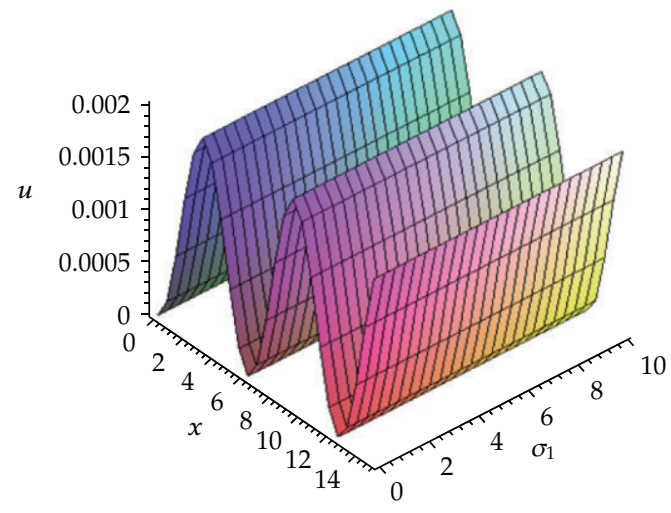

(a)

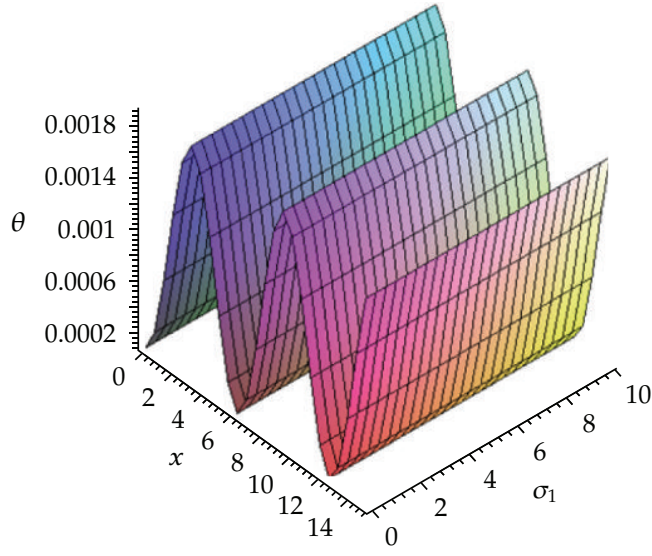

(b)

Figure 7: Variations of the displacement $u$ and temperature $\theta$ for various values of the axis $x$ and magnetic field $\sigma_{1}$ when $t=0.1, \Omega=0.1, \sigma_{2}=0.1$.

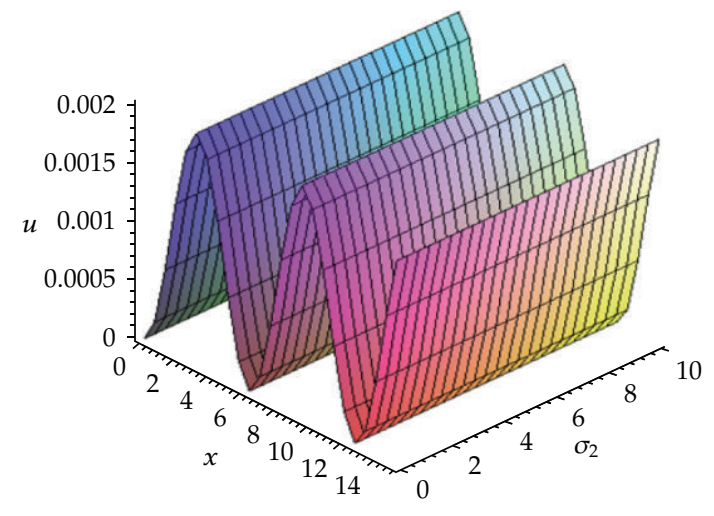

(a)

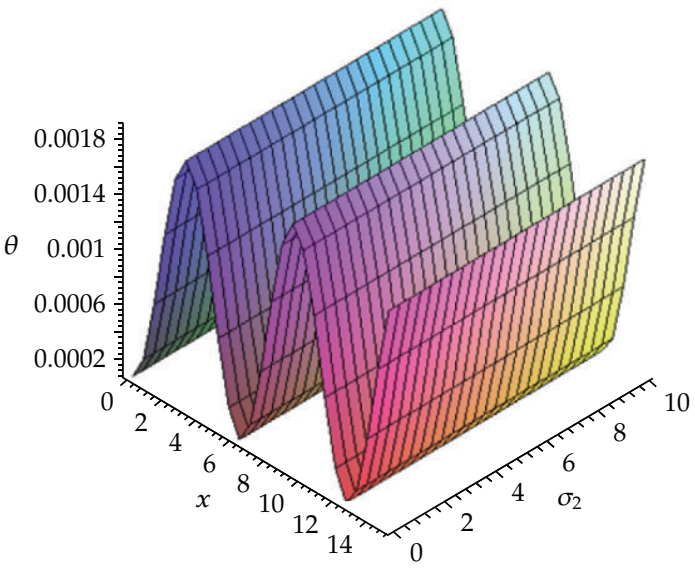

(b)

Figure 8: Variations of the displacement $u$ and temperature $\theta$ for various values of the axis $x$ and magnetic field $\sigma_{2}$ when $t=0.1, \Omega=0.1, \sigma_{1}=0.1$.

\section{Basic Idea of Variational Iteration Method}

Consider the following nonhomogeneous nonlinear system of partial differential equations:

$$
\begin{aligned}
& L_{1} u(x, t)+N_{1}(u(x, t), \theta(x, t))=f(x, t), \\
& L_{2} \theta(x, t)+N_{2}(u(x, t), \theta(x, t))=g(x, t),
\end{aligned}
$$




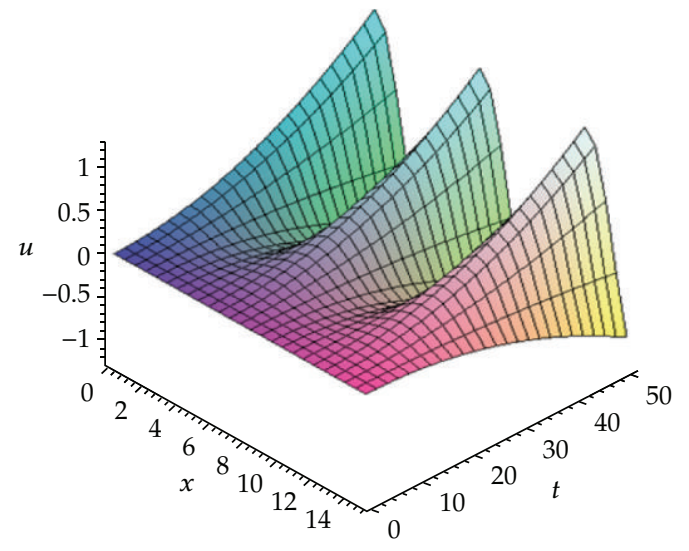

(a)

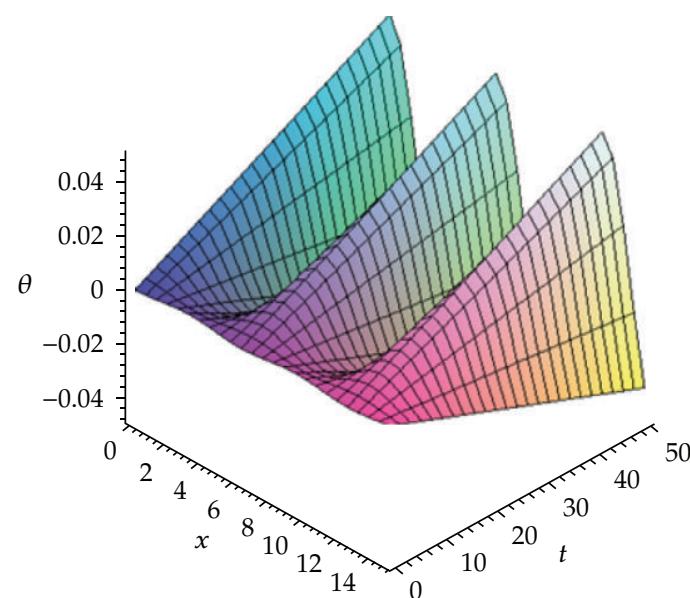

(b)

Figure 9: Variations of the displacement $u$ and temperature $\theta$ for various values of the axis $x$ and time $t$ $\left(u=V_{0}+V_{1}+V_{2}\right.$ and $\left.\theta=\Theta_{0}+\Theta_{1}+\Theta_{2}\right)$ when $\Omega=\sigma_{1}=\sigma_{2}=0$.

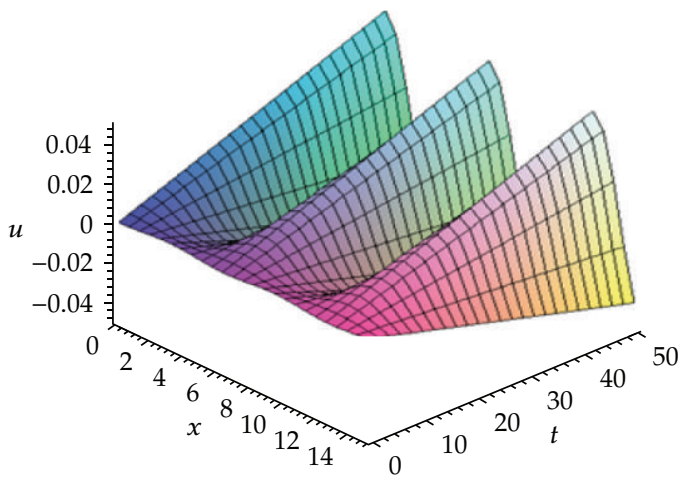

(a)

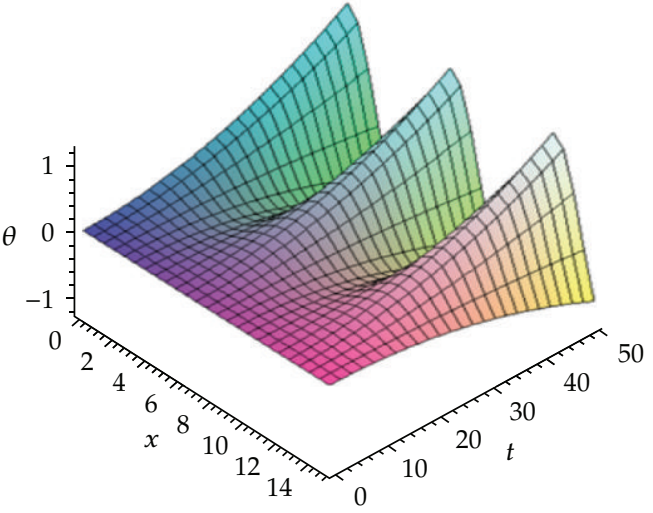

(b)

Figure 10: Variations of the displacement $u$ and temperature $\Theta$ for various values of the axis $x$ and time $t$ $\left(u=V_{0}+V_{1}\right.$ and $\left.\theta=\Theta_{0}+\Theta_{1}\right)$ when $\Omega=\sigma_{1}=\sigma_{2}=0$.

where $L_{1}, L_{2}$ are linear differential operators with respect to time, $N_{1}, N_{2}$ are nonlinear operators, and $f(x, t), g(x, t)$ are given functions. follows:

According to the variational iteration method, we can construct correct functionals as

$$
\begin{aligned}
& u_{n+1}(x, t)=u_{n}(x, t)+\int_{0}^{t} \lambda_{1}(\tau)\left[L_{1} u_{n}(x, \tau)+N_{1}\left(\tilde{u}_{n}(x, \tau), \tilde{\theta}_{n}(x, \tau)\right)-f(x, \tau)\right] d \tau \\
& \theta_{n+1}(x, t)=\theta_{n}(x, t)+\int_{0}^{t} \lambda_{2}(\tau)\left[L_{2} \theta_{n}(x, \tau)+N_{2}\left(\tilde{u}_{n}(x, \tau), \tilde{\theta}_{n}(x, \tau)\right)-g(x, \tau)\right] d \tau
\end{aligned}
$$


where $\lambda_{1}$ and $\lambda_{2}$ are general Lagrange multipliers, which can be identified optimally via variational theory [8-20]. The second term on the right-hand side in (4.3) and (4.4) is called the corrections, and the subscript $n$ denotes the $n$th order approximation, $\tilde{u}_{n}$ and $\tilde{\theta}_{n}$ are restricted variations. We can assume that the above correctional functionals are stationary (i.e., $\delta u_{n+1}=0$ and $\delta \theta_{n+1}=0$ ), then the Lagrange multipliers can be identified. Now we can start with the given initial approximation and by the previous iteration formulas we can obtain the approximate solutions.

\section{Application of the Variational Iteration Method on the Nonlinear Magneto-Thermoelastic with Rotation Equations}

According to the variational iteration method and after some manipulation of (4.3) and (4.4), the correct functionals are as follows:

$$
\begin{gathered}
\begin{aligned}
u_{n+1}(x, t)=u_{n}(x, t)+\int_{0}^{t} \lambda_{1}(\tau)[ & \left(1+\sigma_{1}\right) u_{n, t t}(x, \tau)+\Omega \tilde{u}_{n, t}(x, \tau) \\
& -\tilde{u}_{n, x x}\left(1-\sigma_{2}+2 \gamma \tilde{u}_{n, x}(x, \tau)+3 \delta \tilde{u}_{n, x}^{2}(x, \tau)\right) \\
& \left.-\beta_{1} \tilde{\theta}_{n, x}(x, \tau)-\beta_{2}\left(\tilde{\theta}_{n}(x, \tau) \tilde{u}_{n, x}(x, \tau)\right)_{x}\right] d \tau, \\
\theta_{n+1}(x, t)=\theta_{n}(x, t)+\int_{0}^{t} \lambda_{2}(\tau)[ & \theta_{n, t}(x, \tau)-a \tilde{u}_{n, x t}(x, \tau)-b \tilde{u}_{n, x}(x, \tau) \tilde{u}_{n, x t}(x, \tau) \\
- & \left.\tilde{\theta}_{n, x x}(x, \tau)-\alpha \tilde{u}_{n, x x}(x, \tau) \tilde{\theta}_{n, x}(x, \tau)-\alpha \tilde{u}_{n, x}(x, \tau) \tilde{\theta}_{n, x x}(x, \tau)\right] d \tau,
\end{aligned}
\end{gathered}
$$

where $\tilde{u}_{n}$ and $\tilde{\theta}_{n}$ are considered as a restricted variation, that is, $\delta \tilde{u}_{n+1}=0$ and $\delta \tilde{\theta}_{n+1}=0$. Consequently, the general Lagrange multipliers $\lambda_{1}$ and $\lambda_{2}$ take the following form:

$$
\lambda_{1}(\tau)=\frac{\tau-t}{1+\sigma_{1}}, \quad \lambda_{2}(\tau)=-1
$$

By the substitution of the identified Lagrange multipliers (5.2) into (5.1), we have the following iteration relations:

$$
\begin{aligned}
u_{n+1}(x, t)=u_{n}(x, t)+\int_{0}^{t} \frac{\tau-t}{1+\sigma_{1}}[ & \left(1+\sigma_{1}\right) u_{n, t t}(x, \tau)+\Omega u_{n, t}(x, \tau) \\
& -u_{n, x x}\left(1-\sigma_{2}+2 \gamma u_{n, x}(x, \tau)+3 \delta u_{n, x}^{2}(x, \tau)\right) \\
& \left.-\beta_{1} \theta_{n, x}(x, \tau)-\beta_{2}\left(\theta_{n}(x, \tau) u_{n, x}(x, \tau)\right)_{x}\right] d \tau
\end{aligned}
$$


Mathematical Problems in Engineering

$$
\begin{aligned}
& \theta_{n+1}(x, t)=\theta_{n}(x, t)-\int_{0}^{t}[ \theta_{n}(x, \tau)-a u_{n, x t}(x, \tau)-b u_{n, x}(x, \tau) u_{n, x t}(x, \tau)-\theta_{n, x x}(x, \tau) \\
&\left.-\alpha u_{n, x x}(x, \tau) \theta_{n, x}(x, \tau)-\alpha u_{n, x}(x, \tau) \theta_{n, x x}(x, \tau)\right] d \tau, \quad n \geq 0 .
\end{aligned}
$$

With help of Maple or Mathematica, we get the following results:

$$
\begin{aligned}
& u_{0}=\theta_{0}=A(1-\cos x), \\
& u_{1}=-\frac{t^{2}}{2\left(1+\sigma_{1}\right)}\left[-\beta_{1} \sin x-2 A \gamma \cos x \sin (x)+2 \beta_{2} A \cos ^{2} x-\cos x-3 \delta A^{2} \cos x\right. \\
& \left.-\beta_{2} A+\sigma_{2} \cos x-\beta_{2} A \cos x+3 \delta A^{2} \cos ^{3} x\right]+A(1-\cos x), \\
& \theta_{1}=A[\cos x+2 \alpha A \cos x \sin x] t+A(1-\cos x), \\
& u_{2}=\left\{-\frac{A \cos ^{6}(x)}{6720\left(1+\sigma_{1}\right)^{4}}\left(-19440 A^{5} \delta^{2} \beta_{1} \gamma+5760 A^{5} \delta \beta_{2}^{3}-247860 A^{7} \delta^{3} \beta_{2}-19440 A^{6} \delta^{2} \beta_{2}^{2}\right.\right. \\
& \left.-19440 A^{5} \delta^{2} \beta_{2}-17280 \delta \beta_{2} \gamma^{2} A^{5}+19440 A^{5} \delta^{2} \beta_{2} \sigma_{2}\right) \\
& +\left[-\frac{A \cos ^{2}(x)}{6720\left(1+\sigma_{1}\right)^{4}}\left(-6480 A^{5} \delta \beta_{2}^{2} \gamma+2025 A^{4} \delta \beta_{1} \beta_{2}^{2}+270 A^{3} \delta \beta_{1} \sigma_{2} \beta_{2}+3240 A^{4} \delta^{2} \beta_{1} \sigma_{2}\right.\right. \\
& -38880 A^{6} \delta^{2} \beta_{2} \gamma-8505 A^{6} \delta^{3} \beta_{1}-135 A^{2} \delta \beta_{1} \sigma_{2}^{2}-6480 A^{4} \gamma \beta_{2} \delta \\
& -135 A^{2} \delta \beta_{1}+6480 A^{4} \delta \beta_{2} \gamma \sigma_{2}-3240 A^{5} \delta^{2} \beta_{1} \beta_{2}+270 A^{2} \delta \beta_{1} \sigma_{2} \\
& \left.-270 A^{3} \delta \beta_{1} \beta_{2}-3240 A^{4} \delta^{2} \beta_{1}-2160 A^{4} \delta \beta_{1} \gamma^{2}+45 A^{2} \delta \beta_{1}^{3}\right) \\
& -\frac{A \cos ^{4}(x)}{6720\left(1+\sigma_{1}\right)^{4}}\left(7200 A^{4} \gamma \beta_{2} \delta+118800 A^{6} \delta^{2} \beta_{2} \gamma-4050 A^{4} \delta^{2} \beta_{1} \sigma_{2}+4050 A^{4} \delta^{2} \beta_{1}\right. \\
& +4050 A^{5} \delta^{2} \beta_{1} \beta_{2}+3600 A^{4} \delta \beta_{1} \gamma^{2}-3600 A^{4} \delta \beta_{1} \beta_{2}^{2} \\
& \left.+7200 A^{5} \delta \beta_{2}^{2} \gamma+30375 A^{6} \delta^{3} \beta_{1}-7200 A^{4} \delta \beta_{2} \gamma \sigma_{2}\right) \\
& -\frac{A \cos ^{3}(x)}{6720\left(1+\sigma_{1}\right)^{4}}\left(17280 A^{5} \delta^{2} \beta_{1} \beta_{2}-1440 A^{4} \gamma \beta_{2} \delta-93960 A^{7} \delta^{3} \gamma+16560 A^{5} \delta \beta_{2}^{2} \gamma\right. \\
& +1440 A^{4} \delta \beta_{2} \gamma \sigma_{2}-23760 A^{6} \delta^{2} \beta_{2} \gamma+1440 A^{4} \delta \beta_{1} \beta_{2}^{2}-720 A^{3} \gamma \delta \\
& +1440 A^{3} \delta \beta_{1} \beta_{2}+23760 A^{5} \delta^{2} \gamma \sigma_{2}-720 A^{3} \delta \gamma \sigma_{2}^{2}-5760 A^{5} \delta \gamma^{3} \\
& \left.-23760 A^{5} \gamma \delta^{2}-1440 A^{3} \delta \beta_{1} \sigma_{2} \beta_{2}+720 A^{3} \delta \beta_{1}^{2} \gamma+1440 A^{3} \gamma \delta \sigma_{2}\right) \\
& -\frac{A \cos ^{5}(x)}{6720\left(1+\sigma_{1}\right)^{4}}\left(-19440 A^{5} \delta^{2} \beta_{1} \beta_{2}+189540 A^{7} \delta^{3} \gamma+19440 A^{6} \delta^{2} \beta_{2} \gamma-17280 A^{5} \delta \beta_{2}^{2} \gamma\right. \\
& \left.+19440 A^{5} \gamma \delta^{2}+5760 A^{5} \delta \gamma^{3}-19440 A^{5} \delta^{2} \gamma \sigma_{2}\right)
\end{aligned}
$$




$$
\begin{aligned}
& -\frac{A \cos (x)}{6720\left(1+\sigma_{1}\right)^{4}}\left(-1080 A^{3} \gamma \delta \sigma_{2}+540 A^{3} \gamma \delta+540 A^{3} \delta \gamma \sigma_{2}^{2}-180 A^{3} \delta \beta_{1}^{2} \gamma\right. \\
& -1080 A^{4} \delta \beta_{2} \gamma \sigma_{2}-720 A^{4} \delta \beta_{1} \beta_{2}^{2}-2160 A^{5} \delta^{2} \beta_{1} \beta_{2}+1080 A^{4} \gamma \beta_{2} \delta \\
& +720 A^{3} \delta \beta_{1} \sigma_{2} \beta_{2}+6480 A^{6} \delta^{2} \beta_{2} \gamma-2340 A^{5} \delta \beta_{2}^{2} \gamma-6480 A^{5} \delta^{2} \gamma \sigma_{2} \\
& \left.-720 A^{3} \delta \beta_{1} \beta_{2}+14580 A^{7} \delta^{3} \gamma+1440 A^{5} \delta \gamma^{3}+6480 A^{5} \gamma \delta^{2}\right) \text { e899 } \\
& +\frac{243 A^{8} \delta^{3} \gamma}{141\left(1+\sigma_{1}\right)^{4}} \cos ^{7}(x)-\frac{A \cos ^{6}(x)}{6720\left(1+\sigma_{1}\right)^{4}}\left(-90720 A^{6} \delta^{2} \beta_{2} \gamma-25515 A^{6} \delta^{3} \beta_{1}\right) \\
& -\frac{A}{6720\left(1+\sigma_{1}\right)^{4}}\left(720 A^{5} \delta \beta_{2}^{2} \gamma+45 A^{2} \delta \beta_{1} \sigma_{2}^{2}+405 A^{6} \delta^{3} \beta_{1}+720 A^{4} \gamma \beta_{2} \delta\right. \\
& -720 A^{4} \delta \beta_{2} \gamma \sigma_{2}+90 A^{3} \delta \beta_{1} \beta_{2}+45 A^{4} \delta \beta_{1} \beta_{2}^{2}+2160 A^{6} \delta^{2} \beta_{2} \gamma \\
& +270 A^{5} \delta^{2} \beta_{1} \beta_{2}+45 A^{2} \delta \beta_{1}-90 A^{3} \delta \beta_{1} \sigma_{2} \beta_{2}+270 A^{4} \delta^{2} \beta_{1} \\
& \left.\left.-270 A^{4} \delta^{2} \beta_{1} \sigma_{2}-90 A^{2} \delta \beta_{1} \sigma_{2}+180 A^{4} \delta \beta_{1} \gamma^{2}\right)\right] \sin (x) \\
& -\frac{A \cos ^{5}(x)}{6720\left(1+\sigma_{1}\right)^{4}}\left(204120 A^{8} \delta^{4}-7200 A^{4} \delta \beta_{1} \gamma \beta_{2}+52245 A^{6} \delta^{3}+2025 \delta^{2} A^{4}\right. \\
& -52245 A^{6} \delta^{3} \sigma_{2}+3600 A^{4} \gamma^{2} \delta-80055 A^{6} \delta^{2} \beta_{2}^{2}-3600 A^{5} \delta \beta_{2}^{3} \\
& +3600 \delta \beta_{2} \gamma^{2} A^{5}+52245 A^{7} \delta^{3} \beta_{2}+82080 \delta^{2} \gamma^{2} A^{6}+2025 A^{4} \delta^{2} \sigma_{2}^{2} \\
& +3600 A^{4} \delta \beta_{2}^{2} \sigma_{2}-4050 A^{5} \delta^{2} \beta_{2} \sigma_{2}-4050 A^{4} \delta^{2} \sigma_{2}-2025 A^{4} \delta^{2} \beta_{1}^{2} \\
& \left.+4050 A^{5} \delta^{2} \beta_{2}-3600 A^{4} \delta \beta_{2}^{2}-3600 A^{4} \delta \gamma^{2} \sigma_{2}\right)-\frac{243 A^{8} \delta^{3} \beta_{2} \cos ^{8}(x)}{141\left(1+\sigma_{1}\right)^{4}} \\
& -\frac{A \cos ^{4}(x)}{6720\left(1+\sigma_{1}\right)^{4}}\left(25920 \delta \beta_{2} \gamma^{2} A^{5}+1440 A^{4} \delta \beta_{2}^{2}+166860 A^{7} \delta^{3} \beta_{2}+33480 A^{5} \delta^{2} \beta_{2}\right. \\
& -1440 A^{4} \delta \beta_{2}^{2} \sigma_{2}+1440 A^{3} \gamma \beta_{1} \delta-720 A^{3} \delta \beta_{1}^{2} \beta_{2}+33480 A^{6} \delta^{2} \beta_{2}^{2} \\
& -1440 A^{3} \delta \beta_{1} \gamma \sigma_{2}-7920 A^{5} \delta \beta_{2}^{3}+1440 A^{4} \delta \beta_{1} \gamma \beta_{2}+27000 A^{5} \delta^{2} \beta_{1} \gamma \\
& \left.-33480 A^{5} \delta^{2} \beta_{2} \sigma_{2}-1440 A^{3} \delta \beta_{2} \sigma_{2}+720 A^{3} \delta \beta_{2} \sigma_{2}^{2}+720 \delta \beta_{2} A^{3}\right) \\
& -\frac{A \cos ^{3}(x)}{6720\left(1+\sigma_{1}\right)^{4}}\left(2025 A^{4} \delta^{2} \beta_{1}^{2}+6480 A^{4} \delta^{2} \sigma_{2}+5040 A^{4} \delta \gamma^{2} \sigma_{2}+135 A^{3} \delta \beta_{1}^{2} \beta_{2}\right. \\
& -3240 \delta^{2} A^{4}+7920 A^{4} \delta \beta_{1} \gamma \beta_{2}-70470 A^{8} \delta^{4}-32805 A^{6} \delta^{3} \\
& -4905 A^{4} \delta \beta_{2}^{2} \sigma_{2}+135 A^{2} \delta \beta_{1}^{2}+270 A^{3} \delta \beta_{2} \sigma_{2}+6480 A^{5} \delta^{2} \beta_{2} \sigma_{2} \\
& -135 A^{3} \delta \beta_{2} \sigma_{2}^{2}-135 A^{2} \delta \sigma_{2}^{2}+135 A^{2} \sigma_{2} \delta+45 A^{2} \delta \sigma_{2}^{3}-45 A^{2} \delta \\
& -32805 A^{7} \delta^{3} \beta_{2}-45900 \delta^{2} \gamma^{2} A^{6}+32805 A^{6} \delta^{3} \sigma_{2}+37800 A^{6} \delta^{2} \beta_{2}^{2}
\end{aligned}
$$


Mathematical Problems in Engineering

$$
\begin{aligned}
& -135 \delta \beta_{2} A^{3}+4995 A^{5} \delta \beta_{2}^{3}-135 A^{2} \delta \beta_{1}^{2} \sigma_{2}-6480 A^{5} \delta^{2} \beta_{2}-5040 \delta \beta_{2} \gamma^{2} A^{5} \\
& \left.+4905 A^{4} \delta \beta_{2}^{2}-5040 A^{4} \gamma^{2} \delta-3240 A^{4} \delta^{2} \sigma_{2}^{2}\right)-\frac{6561 A^{9} \delta^{4} \cos ^{9}(x)}{4481\left(1+\sigma_{1}\right)^{4}} \\
& -\frac{A \cos ^{2}(x)}{6720\left(1+\sigma_{1}\right)^{4}}\left(540 A^{3} \delta \beta_{1}^{2} \beta_{2}+1980 A^{5} \delta \beta_{2}^{3}-9180 A^{5} \delta^{2} \beta_{1} \gamma-900 \delta \beta_{2} A^{3}\right. \\
& +1800 A^{4} \delta \beta_{2}^{2} \sigma_{2}-15120 A^{5} \delta^{2} \beta_{2}-10080 \delta \beta_{2} \gamma^{2} A^{5}-15120 A^{6} \delta^{2} \beta_{2}^{2} \\
& +1440 A^{3} \delta \beta_{1} \gamma \sigma_{2}-1440 A^{3} \gamma \beta_{1} \delta-37260 A^{7} \delta^{3} \beta_{2}-1440 A^{4} \delta \beta_{1} \gamma \beta_{2} \\
& \left.+15120 A^{5} \delta^{2} \beta_{2} \sigma_{2}+1800 A^{3} \delta \beta_{2} \sigma_{2}-900 A^{3} \delta \beta_{2} \sigma_{2}^{2}-1800 A^{4} \delta \beta_{2}^{2}\right) \\
& -\frac{A}{6720\left(1+\sigma_{1}\right)^{4}}\left(180 A^{4} \delta \beta_{1} \gamma \beta_{2}+360 A^{4} \delta \beta_{2}^{2}+180 \delta \beta_{2} A^{3}-360 A^{4} \delta \beta_{2}^{2} \sigma_{2}\right. \\
& +1080 A^{6} \delta^{2} \beta_{2}^{2}+1080 A^{5} \delta^{2} \beta_{2}+1620 A^{7} \delta^{3} \beta_{2}-180 A^{3} \delta \beta_{1} \gamma \sigma_{2} \\
& +180 A^{3} \gamma \beta_{1} \delta+180 A^{5} \delta \beta_{2}^{3}+540 A^{5} \delta^{2} \beta_{1} \gamma-1080 A^{5} \delta^{2} \beta_{2} \sigma_{2} \\
& \left.-360 A^{3} \delta \beta_{2} \sigma_{2}+180 A^{3} \delta \beta_{2} \sigma_{2}^{2}+720 \delta \beta_{2} \gamma^{2} A^{5}\right) \\
& -\frac{A \cos ^{7}(x)}{6720\left(1+\sigma_{1}\right)^{4}}\left(45360 A^{6} \delta^{2} \beta_{2}^{2}+25515 A^{6} \delta^{3} \sigma_{2}-25515 A^{6} \delta^{3}-45360 \delta^{2} \gamma^{2} A^{6}\right. \\
& \left.-240570 A^{8} \delta^{4}-25515 A^{7} \delta^{3} \beta_{2}\right) \\
& -\frac{A \cos (x)}{6720\left(1+\sigma_{1}\right)^{4}}\left(-270 A^{4} \delta^{2} \beta_{1}^{2}-2430 A^{4} \delta^{2} \sigma_{2}-1620 A^{4} \delta \gamma^{2} \sigma_{2}-90 A^{3} \delta \beta_{1}^{2} \beta_{2}+1215 \delta^{2} A^{4}\right. \\
& -1440 A^{4} \delta \beta_{1} \gamma \beta_{2}+8505 A^{8} \delta^{4}+6075 A^{6} \delta^{3}+1305 A^{4} \delta \beta_{2}^{2} \sigma_{2} \\
& -90 A^{2} \delta \beta_{1}^{2}-270 A^{3} \delta \beta_{2} \sigma_{2}-2430 A^{5} \delta^{2} \beta_{2} \sigma_{2}+135 A^{3} \delta \beta_{2} \sigma_{2}^{2} \\
& +135 A^{2} \delta \sigma_{2}^{2}-135 A^{2} \sigma_{2} \delta-45 A^{2} \delta \sigma_{2}^{3}+45 A^{2} \delta+6075 A^{7} \delta^{3} \beta_{2} \\
& +8100 \delta^{2} \gamma^{2} A^{6}-6075 A^{6} \delta^{3} \sigma_{2}-3105 A^{6} \delta^{2} \beta_{2}^{2}+135 \delta \beta_{2} A^{3} \\
& -1395 A^{5} \delta \beta_{2}^{3}+90 A^{2} \delta \beta_{1}^{2} \sigma_{2}+2430 A^{5} \delta^{2} \beta_{2}+1620 \delta \beta_{2} \gamma^{2} A^{5} \\
& \left.\left.-1305 A^{4} \delta \beta_{2}^{2}+1620 A^{4} \gamma^{2} \delta+1215 A^{4} \delta^{2} \sigma_{2}^{2}\right)\right\} t^{8} \\
& +\left\{-\frac{A \cos ^{6}(x)}{6720\left(1+\sigma_{1}\right)^{4}}\left(72576 A^{5} \delta^{2} \beta_{2} \sigma_{1}+72576 A^{5} \delta^{2} \beta_{2}\right)\right. \\
& +\left[-\frac{A \cos ^{5}(x)}{6720\left(1+\sigma_{1}\right)^{4}}\left(-99792 A^{5} \gamma \delta^{2} \sigma_{1}-99792 A^{5} \gamma \delta^{2}\right)\right. \\
& -\frac{A \cos ^{2}(x)}{6720\left(1+\sigma_{1}\right)^{4}}\left(1008 A^{3} \delta \beta_{1} \beta_{2}+1344 A^{2} \gamma^{2} \beta_{1}-1344 A^{2} \gamma \beta_{2} \sigma_{2} \sigma_{1}-1008 A^{2} \delta \beta_{1} \sigma_{2}\right.
\end{aligned}
$$




$$
\begin{aligned}
& +1344 A^{3} \gamma \beta_{2}^{2}+1344 A^{2} \gamma \beta_{2} \sigma_{1}+1344 A^{3} \gamma \beta_{2}^{2} \sigma_{1}+1344 A^{2} \gamma \beta_{2} \\
& -1008 A^{2} \delta \beta_{1} \sigma_{1} \sigma_{2}+1344 A^{2} \gamma^{2} \beta_{1} \sigma_{1}+12096 A^{4} \delta^{2} \beta_{1} \\
& +1008 A^{3} \delta \beta_{1} \sigma_{1} \beta_{2}+12096 A^{4} \delta^{2} \beta_{1} \sigma_{1}-1344 A^{2} \gamma \beta_{2} \sigma_{2} \\
& +40320 A^{4} \gamma \beta_{2} \delta \sigma_{1}+40320 A^{4} \gamma \beta_{2} \delta+1008 A^{2} \delta \beta_{1} \\
& \left.+1008 A^{2} \delta \beta_{1} \sigma_{1}\right) \\
& -\frac{A}{6720\left(1+\sigma_{1}\right)^{4}}\left(-448 A^{2} \gamma \beta_{2}+448 A^{2} \gamma \beta_{2} \sigma_{2} \sigma_{1}-448 A^{2} \gamma \beta_{2} \sigma_{1}-448 A^{3} \gamma \beta_{2}^{2} \sigma_{1}\right. \\
& +448 A^{2} \gamma \beta_{2} \sigma_{2}+336 A^{2} \delta \beta_{1} \sigma_{2}-1008 A^{4} \delta^{2} \beta_{1}-336 A^{3} \delta \beta_{1} \beta_{2} \\
& -336 A^{2} \delta \beta_{1}-336 A^{3} \delta \beta_{1} \sigma_{1} \beta_{2}+336 A^{2} \delta \beta_{1} \sigma_{1} \sigma_{2}-336 A^{2} \delta \beta_{1} \sigma_{1} \\
& -4032 A^{4} \gamma \beta_{2} \delta-224 A^{2} \gamma^{2} \beta_{1}-448 A^{3} \gamma \beta_{2}^{2}-4032 A^{4} \gamma \beta_{2} \delta \sigma_{1} \\
& \left.-224 A^{2} \gamma^{2} \beta_{1} \sigma_{1}-1008 A^{4} \delta^{2} \beta_{1} \sigma_{1}\right) \\
& -\frac{A \cos (x)}{6720\left(1+\sigma_{1}\right)^{4}}\left(2688 A^{3} \delta \beta_{1} \beta_{2}+1680 A^{3} \gamma \beta_{2}^{2} \sigma_{1}-1792 A^{3} \gamma^{3}+2688 A^{3} \delta \beta_{1} \sigma_{1} \beta_{2}\right. \\
& +6720 A^{3} \gamma \delta \sigma_{2} \sigma_{1}-6720 A^{3} \gamma \delta \sigma_{1}+6720 A^{3} \gamma \delta \sigma_{2}-31248 A^{5} \gamma \delta^{2} \\
& -224 A^{2} \gamma \beta_{2} \sigma_{1}+224 A^{2} \gamma \beta_{2} \sigma_{2} \sigma_{1}+1680 A^{3} \gamma \beta_{2}^{2}+224 A^{2} \gamma \beta_{2} \sigma_{2} \\
& -1792 A^{3} \gamma^{3} \sigma_{1}-6720 A^{3} \gamma \delta-224 A^{2} \gamma \beta_{2}-112 A \gamma \\
& +112 A \gamma \beta_{1}^{2}+112 A \gamma \beta_{1}^{2} \sigma_{1}-112 A \gamma \sigma_{2}^{2} \sigma_{1}+224 A \gamma \sigma_{2} \sigma_{1} \\
& -112 A \gamma \sigma_{2}^{2}+224 A \gamma \sigma_{2}-112 A \gamma \sigma_{1}-6720 A^{4} \gamma \beta_{2} \delta \sigma_{1} \\
& \left.-31248 A^{5} \gamma \delta^{2} \sigma_{1}-6720 A^{4} \gamma \beta_{2} \delta\right) \\
& -\frac{A \cos ^{3}(x)}{6720 /\left(1+\sigma_{1}\right)^{4}}\left(-3584 A^{3} \gamma \beta_{2}^{2} \sigma_{1}+9408 A^{4} \gamma \beta_{2} \delta \sigma_{1}+118944 A^{5} \gamma \delta^{2}+9408 A^{4} \gamma \beta_{2} \delta\right. \\
& +3584 A^{3} \gamma^{3} \sigma_{1}+9408 A^{3} \gamma \delta+9408 A^{3} \gamma \delta \sigma_{1}+118944 A^{5} \gamma \delta^{2} \sigma_{1} \\
& -3584 A^{3} \gamma \beta_{2}^{2}-5376 A^{3} \delta \beta_{1} \beta_{2}+3584 A^{3} \gamma^{3}-9408 A^{3} \gamma \delta \sigma_{2} \\
& \left.-5376 A^{3} \delta \beta_{1} \sigma_{1} \beta_{2}-9408 A^{3} \gamma \delta \sigma_{2} \sigma_{1}\right) \\
& -\frac{A \cos ^{4}(x)}{6720\left(1+\sigma_{1}\right)^{4}}\left(-15120 A^{4} \delta^{2} \beta_{1}-47040 A^{4} \gamma \beta_{2} \delta-15120 A^{4} \delta^{2} \beta_{1} \sigma_{1}\right. \\
& \left.\left.-47040 A^{4} \gamma \beta_{2} \delta \sigma_{1}\right)\right] \sin (x) \\
& -\frac{A \cos ^{7}(x)}{6720\left(1+\sigma_{1}\right)^{4}}\left(95256 A^{6} \delta^{3} \sigma_{1}+95256 A^{6} \delta^{3}\right)
\end{aligned}
$$




$$
\begin{aligned}
& -\frac{A \cos ^{3}(x)}{6720\left(1+\sigma_{1}\right)^{4}}\left(-24192 A^{4} \delta^{2} \sigma_{2}-24192 A^{4} \delta^{2} \sigma_{1} \sigma_{2}+24192 \delta^{2} A^{4}-1344 A^{2} \gamma \beta_{1} \beta_{2} \sigma_{1}\right. \\
& +122472 A^{6} \delta^{3}+1008 A^{3} \beta_{2} \delta \sigma_{1}-1344 A^{2} \gamma \beta_{1} \beta_{2}-18312 A^{4} \delta \beta_{2}^{2} \sigma_{1} \\
& +1344 A^{3} \gamma^{2} \beta_{2} \sigma_{1}-504 A^{2} \delta \beta_{1}^{2}+1344 A^{2} \gamma^{2} \sigma_{1}-1008 A^{3} \delta \beta_{2} \sigma_{2} \\
& +24192 A^{5} \delta^{2} \beta_{2} \sigma_{1}+504 A^{2} \delta \sigma_{1}+24192 A^{4} \delta^{2} \sigma_{1}+54024 A^{4} \gamma^{2} \delta \sigma_{1} \\
& -1344 A^{2} \gamma^{2} \sigma_{2}+504 A^{2} \delta \sigma_{2}^{2}-1008 A^{2} \sigma_{2} \delta+1008 A^{2} \sigma_{2} \delta \sigma_{1} \\
& +504 A^{2} \delta-1008 A^{3} \delta \beta_{2} \sigma_{1} \sigma_{2}-1344 A^{2} \gamma^{2} \sigma_{2} \sigma_{1}-122472 A^{6} \delta^{3} \sigma_{1} \\
& +1008 \delta \beta_{2} A^{3}+1344 A^{2} \gamma^{2}+504 A^{2} \delta \sigma_{1} \sigma_{2}^{2}-504 A^{2} \delta \beta_{1}^{2} \sigma_{1} \\
& \left.+24192 A^{5} \delta^{2} \beta_{2}-18312 A^{4} \delta \beta_{2}^{2}+45024 A^{4} \gamma^{2} \delta+1344 A^{3} \gamma^{2} \beta_{2}\right) \\
& -\frac{A \cos ^{5}(x)}{6720\left(1+\sigma_{1}\right)^{4}}\left(-195048 A^{6} \delta^{3}+15120 A^{4} \delta^{2} \sigma_{1} \sigma_{2}+15120 A^{4} \delta^{2} \sigma_{2}-195048 A^{6} \delta^{3} \sigma_{1}\right. \\
& -15120 A^{5} \delta^{2} \beta_{2}+13440 A^{4} \delta \beta_{2}^{2}-33600 A^{4} \gamma^{2} \delta \sigma_{1} \\
& -33600 A^{4} \gamma^{2} \delta-15120 \delta^{2} A^{4}-15120 A^{4} \delta^{2} \sigma_{1}-15120 A^{5} \delta^{2} \beta_{2} \sigma_{1} \\
& \left.+13440 A^{4} \delta \beta_{2}^{2} \sigma_{1}\right) \\
& -\frac{A}{6720\left(1+\sigma_{1}\right)^{4}}\left(-112 A \gamma \beta_{1} \sigma_{1}-1344 \delta \beta_{2} A^{3}-112 A^{2} \gamma \beta_{1} \beta_{2}-1008 A^{3} \gamma \beta_{1} \delta \sigma_{1}\right. \\
& +112 A \gamma \beta_{1} \sigma_{2} \sigma_{1}+1344 A^{3} \delta \beta_{2} \sigma_{1} \sigma_{2}-896 A^{3} \gamma^{2} \beta_{2}-112 A^{2} \gamma \beta_{1} \beta_{2} \sigma_{1} \\
& -1344 A^{3} \beta_{2} \delta \sigma_{1}-1344 A^{4} \delta \beta_{2}^{2} \sigma_{1}-1008 A^{3} \gamma \beta_{1} \delta-112 A \gamma \beta_{1} \\
& +112 A \gamma \beta_{1} \sigma_{2}-896 A^{3} \gamma^{2} \beta_{2} \sigma_{1}-4032 A^{5} \delta^{2} \beta_{2}-1344 A^{4} \delta \beta_{2}^{2} \\
& \left.+1344 A^{3} \delta \beta_{2} \sigma_{2}-4032 A^{5} \delta^{2} \beta_{2} \sigma_{1}\right) \\
& -\frac{A \cos ^{4}(x)}{6720\left(1+\sigma_{1}\right)^{4}}\left(-7168 A^{3} \gamma^{2} \beta_{2}-5376 A^{4} \delta \beta_{2}^{2} \sigma_{1}-9408 A^{3} \gamma \beta_{1} \delta-9408 A^{3} \gamma \beta_{1} \delta \sigma_{1}\right. \\
& -5376 A^{3} \beta_{2} \delta \sigma_{1}-7168 A^{3} \gamma^{2} \beta_{2} \sigma_{1}-5376 \delta \beta_{2} A^{3}-124992 A^{5} \delta^{2} \beta_{2} \\
& \left.-5376 A^{4} \delta \beta_{2}^{2}+5376 A^{3} \delta \beta_{2} \sigma_{2}+5376 A^{3} \delta \beta_{2} \sigma_{1} \sigma_{2}-124992 A^{5} \delta^{2} \beta_{2} \sigma_{1}\right) \\
& -\frac{A \cos (x)}{6720\left(1+\sigma_{1}\right)^{4}}\left(9072 A^{4} \delta^{2} \sigma_{2}+9072 A^{4} \delta^{2} \sigma_{1} \sigma_{2}-9072 \delta^{2} A^{4}+896 A^{2} \gamma \beta_{1} \beta_{2} \sigma_{1}\right. \\
& -22680 A^{6} \delta^{3}-1008 A^{3} \beta_{2} \delta \sigma_{1}+896 A^{2} \gamma \beta_{1} \beta_{2}+4872 A^{4} \delta \beta_{2}^{2} \sigma_{1} \\
& -1120 A^{3} \gamma^{2} \beta_{2} \sigma_{1}+336 A^{2} \delta \beta_{1}^{2}-1120 A^{2} \gamma^{2} \sigma_{1}+1008 A^{3} \delta \beta_{2} \sigma_{2} \\
& -9072 A^{5} \delta^{2} \beta_{2} \sigma_{1}-504 A^{2} \delta \sigma_{1}-9072 A^{4} \delta^{2} \sigma_{1}-13440 A^{4} \gamma^{2} \delta \sigma_{1}
\end{aligned}
$$




$$
\begin{aligned}
& +1120 A^{2} \gamma^{2} \sigma_{2}-504 A^{2} \delta \sigma_{2}^{2}+1008 A^{2} \sigma_{2} \delta+1008 A^{2} \sigma_{2} \delta \sigma_{1} \\
& -504 A^{2} \delta+1008 A^{3} \delta \beta_{2} \sigma_{1} \sigma_{2}+1120 A^{2} \gamma^{2} \sigma_{2} \sigma_{1}-22680 A^{6} \delta^{3} \sigma_{1} \\
& -1008 \delta \beta_{2} A^{3}-1120 A^{2} \gamma^{2}-504 A^{2} \delta \sigma_{1} \sigma_{2}^{2}+336 A^{2} \delta \beta_{1}^{2} \sigma_{1} \\
& \left.-9072 A^{5} \delta^{2} \beta_{2}+4872 A^{4} \delta \beta_{2}^{2}-13440 A^{4} \gamma^{2} \delta-1120 A^{3} \gamma^{2} \beta_{2}\right) \\
& -\frac{A \cos ^{2}(x)}{6720\left(1+\sigma_{1}\right)^{4}}\left(-224 A \gamma \beta_{1} \sigma_{2} \sigma_{1}+224 A \gamma \beta_{1} \sigma_{1}+224 A^{2} \gamma \beta_{1} \beta_{2}+56448 A^{5} \delta^{2} \beta_{2}\right. \\
& +6720 A^{4} \delta \beta_{2}^{2}+9072 A^{3} \gamma \beta_{1} \delta \sigma_{1}+224 A \gamma \beta_{1}+6720 \delta \beta_{2} A^{3} \\
& +9072 A^{3} \gamma \beta_{1} \delta+224 A^{2} \gamma \beta_{1} \beta_{2} \sigma_{1}+7168 A^{3} \gamma^{2} \beta_{2}+56448 A^{5} \delta^{2} \beta_{2} \sigma_{1} \\
& +6720 A^{3} \beta_{2} \delta \sigma_{1}+7168 A^{3} \gamma^{2} \beta_{2} \sigma_{1}-6720 A^{3} \delta \beta_{2} \sigma_{1} \sigma_{2} \\
& \left.\left.-224 A \gamma \beta_{1} \sigma_{2}+6720 A^{4} \delta \beta_{2}^{2} \sigma_{1}-6720 A^{3} \delta \beta_{2} \sigma_{2}\right)\right\} t^{6} \\
& +\left\{-\frac{A \cos ^{3}(x)}{6720\left(1+\sigma_{1}\right)^{4}}\left(-1008 A^{2} \beta_{2} \beta_{1} \alpha \sigma_{1}^{2}-4032 A^{2} \beta_{2}^{2} \sigma_{1}-1008 A^{2} \beta_{2} \beta_{1} \alpha-2016 A^{2} \beta_{2} \beta_{1} \alpha \sigma_{1}\right.\right. \\
& \left.-2016 A^{2} \beta_{2}^{2}-2016 A^{2} \beta_{2}^{2} \sigma_{1}^{2}\right) \\
& -\frac{A \cos (x)}{6720\left(1+\sigma_{1}\right)^{4}}\left(1344 A^{2} \beta_{2}^{2} \sigma_{1}^{2}+672 A^{2} \alpha \beta_{2} \beta_{1}+1344 A^{2} \beta_{2}^{2}+2688 A^{2} \beta_{2}^{2} \sigma_{1}\right. \\
& \left.+1344 A^{2} \beta_{2} \beta_{1} \alpha \sigma_{1}+672 A^{2} \beta_{2} \beta_{1} \alpha \sigma_{1}^{2}\right) \\
& -\frac{A}{6720\left(1+\sigma_{1}\right)^{4}}\left(-504 \delta \beta_{2} A^{3}-336 A^{2} \beta_{2}^{2} \sigma_{1}-168 A \beta_{2} \sigma_{1}^{2}+336 A \beta_{2} \sigma_{2} \sigma_{1}\right. \\
& +168 A \beta_{2} \sigma_{2}-1008 A^{3} \beta_{2} \delta \sigma_{1}-168 \beta_{2} A-504 A^{3} \beta_{2} \delta \sigma_{1}^{2} \\
& -168 A^{2} \beta_{2}^{2}-672 A^{3} \beta_{2} \gamma \alpha \sigma_{1}^{2}-168 A^{2} \beta_{2}^{2} \sigma_{1}^{2}-336 A \beta_{2} \sigma_{1} \\
& \left.+168 A \beta_{2} \sigma_{2} \sigma_{1}^{2}-672 A^{3} \beta_{2} \gamma \alpha-1344 A^{3} \beta_{2} \gamma \alpha \sigma_{1}\right) \\
& -\frac{A \cos ^{2}(x)}{6720\left(1+\sigma_{1}\right)^{4}}\left(5544 A^{3} \beta_{2} \delta \sigma_{1}^{2}+5376 A^{3} \beta_{2} \gamma \alpha \sigma_{1}^{2}-672 A \beta_{2} \sigma_{2} \sigma_{1}-336 A \beta_{2} \sigma_{2}\right. \\
& +336 \beta_{2} A+672 A^{2} \beta_{2}^{2} \sigma_{1}+336 A^{2} \beta_{2}^{2}+5376 A^{3} \beta_{2} \gamma \alpha \\
& +672 A \beta_{2} \sigma_{1}-336 A \beta_{2} \sigma_{2} \sigma_{1}^{2}+336 A \beta_{2} \sigma_{1}^{2}+10752 A^{3} \beta_{2} \gamma \alpha \sigma_{1} \\
& \left.+336 A^{2} \beta_{2}^{2} \sigma_{1}^{2}+5544 \delta \beta_{2} A^{3}+11088 A^{3} \beta_{2} \delta \sigma_{1}\right) \\
& +\left[-\frac{A \cos (x)}{6720\left(1+\sigma_{1}\right)^{4}}\left(672 \beta_{2} A \beta_{1} \sigma_{1}+336 \beta_{2} A \beta_{1} \sigma_{1}^{2}+2688 A^{3} \beta_{2}^{2} \alpha \sigma_{1}^{2}\right.\right. \\
& \left.+2688 A^{3} \beta_{2}^{2} \alpha+336 \beta_{2} A \beta_{1}+5376 A^{3} \beta_{2}^{2} \alpha \sigma_{1}\right)
\end{aligned}
$$




$$
\begin{aligned}
& -\frac{A \cos ^{2}(x)}{6720\left(1+\sigma_{1}\right)^{4}}\left(2016 A^{2} \gamma \beta_{2}+24192 A^{4} \beta_{2} \delta \alpha \sigma_{1}+2016 A^{2} \gamma \beta_{2} \sigma_{1}^{2}+2016 A^{2} \beta_{2} \sigma_{1} \alpha\right. \\
& +1008 A^{3} \beta_{2}^{2} \alpha+12096 A^{4} \beta_{2} \delta \alpha \sigma_{1}^{2}+1008 A^{3} \beta_{2}^{2} \alpha \sigma_{1}^{2}+4032 A^{2} \gamma \beta_{2} \sigma_{1} \\
& -1008 A^{2} \beta_{2} \sigma_{2} \alpha \sigma_{1}^{2}+2016 A^{3} \beta_{2}^{2} \alpha \sigma_{1}-2016 A^{2} \beta_{2} \sigma_{2} \alpha \sigma_{1} \\
& \left.+12096 A^{4} \beta_{2} \delta \alpha+1008 A^{2} \beta_{2} \sigma_{1}^{2} \alpha+1008 A^{2} \beta_{2} \alpha-1008 A^{2} \beta_{2} \sigma_{2} \alpha\right) \\
& -\frac{A}{6720\left(1+\sigma_{1}\right)^{4}}\left(-336 A^{2} \gamma \beta_{2}-336 A^{2} \beta_{2} \alpha-2016 A^{4} \beta_{2} \delta \alpha \sigma_{1}-1008 A^{4} \beta_{2} \delta \alpha \sigma_{1}^{2}\right. \\
& -1008 A^{4} \beta_{2} \delta \alpha-336 A^{2} \beta_{2} \sigma_{1}^{2} \alpha-672 A^{2} \gamma \beta_{2} \sigma_{1}+336 A^{2} \beta_{2} \sigma_{2} \alpha \\
& -336 A^{3} \beta_{2}^{2} \alpha \sigma_{1}^{2}+672 A^{2} \beta_{2} \sigma_{2} \alpha \sigma_{1}+336 A^{2} \beta_{2} \sigma_{2} \alpha \sigma_{1}^{2}-336 A^{3} \beta_{2}^{2} \alpha \\
& \left.-672 A^{3} \beta_{2}^{2} \alpha \sigma_{1}-336 A^{2} \gamma \beta_{2} \sigma_{1}^{2}-672 A^{2} \beta_{2} \sigma_{1} \alpha\right) \\
& -\frac{A \cos ^{4}(x)}{6720\left(1+\sigma_{1}\right)^{4}}\left(-30240 A^{4} \beta_{2} \delta \alpha \sigma_{1}-15120 A^{4} \beta_{2} \delta \alpha-15120 A^{4} \beta_{2} \delta \alpha \sigma_{1}^{2}\right) \\
& \left.-\frac{A \cos ^{3}(x)}{6720\left(1+\sigma_{1}\right)^{4}}\left(-5376 A^{3} \beta_{2}^{2} \alpha \sigma_{1}^{2}-5376 A^{3} \beta_{2}^{2} \alpha-10752 A^{3} \beta_{2}^{2} \alpha \sigma_{1}\right)\right] \sin (x) \\
& -\frac{A \cos ^{4}(x)}{6720\left(1+\sigma_{1}\right)^{4}}\left(-5376 A^{3} \beta_{2} \gamma \alpha-12096 A^{3} \beta_{2} \delta \sigma_{1}-6048 \delta \beta_{2} A^{3}-6048 A^{3} \beta_{2} \delta \sigma_{1}^{2}\right. \\
& \left.\left.-10752 A^{3} \beta_{2} \gamma \alpha \sigma_{1}-5376 A^{3} \beta_{2} \gamma \alpha \sigma_{1}^{2}\right)\right\} t^{5} \\
& +\left\{-\frac{\cos ^{2}(x)}{6720\left(1+\sigma_{1}\right)^{4}}\left(5600 A \beta_{2} \sigma_{2} \sigma_{1}-26040 A^{3} \beta_{2} \delta \sigma_{1}^{2}-5600 A \beta_{2} \sigma_{1}-2800 A^{2} \beta_{2}^{2}\right.\right. \\
& -2800 A^{2} \beta_{2}^{2} \sigma_{1}^{2}-2800 \beta_{2} A-52080 A^{3} \beta_{2} \delta \sigma_{1}+2800 A \beta_{2} \sigma_{2} \sigma_{1}^{2} \\
& -26040 \delta \beta_{2} A^{3}-2240 A \gamma \beta_{1} \sigma_{1}-1120 A \gamma \beta_{1}-5600 A^{2} \beta_{2}^{2} \sigma_{1} \\
& \left.-2800 A \beta_{2} \sigma_{1}^{2}-1120 A \gamma \beta_{1} \sigma_{1}^{2}+2800 A \beta_{2} \sigma_{2}\right) \\
& -\frac{A \cos (x)}{6720\left(1+\sigma_{1}\right)^{4}}\left(280+560 \sigma_{1}-560 \sigma_{2}+22680 \delta^{2} A^{4}-560 \sigma_{2} \sigma_{1}^{2}+560 \beta_{2} A\right. \\
& +1120 A \beta_{2} \sigma_{1}-560 A \beta_{2} \sigma_{2}+560 A \beta_{2} \sigma_{1}^{2}-1960 A^{2} \beta_{2}^{2} \sigma_{1}^{2}+8400 A^{3} \beta_{2} \delta \sigma_{1}^{2} \\
& +16800 A^{3} \beta_{2} \delta \sigma_{1}+5600 A^{2} \gamma^{2} \sigma_{1}^{2}+560 \sigma_{2}^{2} \sigma_{1}+280 \sigma_{2}^{2} \sigma_{1}^{2}-560 A \beta_{2} \sigma_{2} \sigma_{1}^{2} \\
& +280 \sigma_{2}^{2}+11200 A^{2} \gamma^{2} \sigma_{1}+16800 A^{2} \delta \sigma_{1}+45360 A^{4} \delta^{2} \sigma_{1}+22680 A^{4} \delta^{2} \sigma_{1}^{2} \\
& -8400 A^{2} \sigma_{2} \delta-8400 A^{2} \sigma_{2} \delta \sigma_{1}^{2}-16800 A^{2} \sigma_{2} \delta \sigma_{1}+8400 A^{2} \delta \sigma_{1}^{2}+280 \sigma_{1}^{2} \\
& +8400 A^{2} \delta-1120 \sigma_{2} \sigma_{1}+8400 \delta \beta_{2} A^{3}+5600 A^{2} \gamma^{2} \\
& \left.-1960 A^{2} \beta_{2}^{2}-1120 A \beta_{2} \sigma_{2} \sigma_{1}-3920 A^{2} \beta_{2}^{2} \sigma_{1}\right)
\end{aligned}
$$




$$
\begin{aligned}
& -\frac{A \cos ^{4}(x)}{6720\left(1+\sigma_{1}\right)^{4}}\left(23520 A^{3} \beta_{2} \delta \sigma_{1}^{2}+47040 A^{3} \beta_{2} \delta \sigma_{1}+23520 \delta \beta_{2} A^{3}\right) \\
& -\frac{A \cos ^{3}(x)}{6720\left(1+\sigma_{1}\right)^{4}}\left(10080 A^{2} \sigma_{2} \delta-6720 A^{2} \gamma^{2}-13440 A^{2} \gamma^{2} \sigma_{1}+10080 A^{2} \sigma_{2} \delta \sigma_{1}^{2}\right. \\
& +3360 A^{2} \beta_{2}^{2}-10080 A^{2} \delta \sigma_{1}^{2}-60480 \delta^{2} A^{4}-10080 A^{2} \delta \\
& -6720 A^{2} \gamma^{2} \sigma_{1}^{2}-120960 A^{4} \delta^{2} \sigma_{1}-20160 A^{3} \beta_{2} \delta \sigma_{1}-10080 \delta \beta_{2} A^{3} \\
& -20160 A^{2} \delta \sigma_{1}+20160 A^{2} \sigma_{2} \delta \sigma_{1}+6720 A^{2} \beta_{2}^{2} \sigma_{1}+3360 A^{2} \beta_{2}^{2} \sigma_{1}^{2} \\
& \left.-60480 A^{4} \delta^{2} \sigma_{1}^{2}-10080 A^{3} \beta_{2} \delta \sigma_{1}^{2}\right) \\
& +\left[-\frac{A \cos (x)}{6720\left(1+\sigma_{1}\right)^{4}}\left(-560 \beta_{2} A \beta_{1}+23520 A^{3} \gamma \delta \sigma_{1}^{2}+23520 A^{3} \gamma \delta-1120 \beta_{2} A \beta_{1} \sigma_{1}\right.\right. \\
& +3360 A \gamma \sigma_{1}^{2}-3360 A \gamma \sigma_{2} \sigma_{1}^{2}+6720 A \gamma \sigma_{1}-560 \beta_{2} A \beta_{1} \sigma_{1}^{2} \\
& +47040 A^{3} \gamma \delta \sigma_{1}+3360 A^{2} \gamma \beta_{2} \sigma_{1}^{2}-3360 A \gamma \sigma_{2}+3360 A^{2} \gamma \beta_{2} \\
& \left.+6720 A^{2} \gamma \beta_{2} \sigma_{1}-6720 A \gamma \sigma_{2} \sigma_{1}+3360 A \gamma\right) \\
& -\frac{A}{6720\left(1+\sigma_{1}\right)^{4}}\left(560 \beta_{1} \sigma_{1}-280 \sigma_{2} \beta_{1}-280 \sigma_{2} \beta_{1} \sigma_{1}^{2}-560 \sigma_{2} \beta_{1} \sigma_{1}+280 \beta_{1}\right. \\
& +1680 A^{2} \delta \beta_{1} \sigma_{1}+280 \beta_{2} A \beta_{1} \sigma_{1}^{2}+560 \beta_{2} A \beta_{1} \sigma_{1}+2800 A^{2} \gamma \beta_{2} \\
& +280 \beta_{1} \sigma_{1}^{2}+2800 A^{2} \gamma \beta_{2} \sigma_{1}^{2}+5600 A^{2} \gamma \beta_{2} \sigma_{1}+840 A^{2} \delta \beta_{1} \sigma_{1}^{2} \\
& \left.+280 \beta_{2} A \beta_{1}+840 A^{2} \delta \beta_{1}\right) \\
& -\frac{A \cos ^{3}(x)}{6720\left(1+\sigma_{1}\right)^{4}}\left(-33600 A^{3} \gamma \delta-67200 A^{3} \gamma \delta \sigma_{1}-33600 A^{3} \gamma \delta \sigma_{1}^{2}\right) \\
& -\frac{A \cos ^{2}(x)}{6720\left(1+\sigma_{1}\right)^{4}}\left(-10080 A^{2} \gamma \beta_{2}-20160 A^{2} \gamma \beta_{2} \sigma_{1}-5040 A^{2} \delta \beta_{1} \sigma_{1}-2520 A^{2} \delta \beta_{1} \sigma_{1}^{2}\right. \\
& \left.\left.-10080 A^{2} \gamma \beta_{2} \sigma_{1}^{2}-2520 A^{2} \delta \beta_{1}\right)\right] \sin (x) \\
& -\frac{A \cos ^{5}(x)}{6720\left(1+\sigma_{1}\right)^{4}}\left(37800 \delta^{2} A^{4}+37800 A^{4} \delta^{2} \sigma_{1}^{2}+75600 A^{4} \delta^{2} \sigma_{1}\right) \\
& -\frac{A}{6720\left(1+\sigma_{1}\right)^{4}}\left(4200 A^{3} \beta_{2} \delta \sigma_{1}^{2}+2800 A^{2} \beta_{2}^{2} \sigma_{1}-1400 A \beta_{2} \sigma_{2} \sigma_{1}^{2}+1400 A \beta_{2} \sigma_{1}^{2}\right. \\
& -2800 A \beta_{2} \sigma_{2} \sigma_{1}+1400 \beta_{2} A+1400 A^{2} \beta_{2}^{2} \sigma_{1}^{2}+560 A \gamma \beta_{1} \sigma_{1}^{2} \\
& +1400 A^{2} \beta_{2}^{2}+8400 A^{3} \beta_{2} \delta \sigma_{1}+4200 \delta \beta_{2} A^{3}-1400 A \beta_{2} \sigma_{2} \\
& \left.\left.+2800 A \beta_{2} \sigma_{1}+1120 A \gamma \beta_{1} \sigma_{1}+560 A \gamma \beta_{1}\right)\right\} t^{4}
\end{aligned}
$$




$$
\begin{aligned}
& +\left\{\left[-\frac{A \cos (x)}{6720\left(1+\sigma_{1}\right)^{4}}\left(2240 \Omega A \gamma+4480 \Omega A \gamma \sigma_{1}+2240 \Omega A \gamma \sigma_{1}^{2}\right)\right.\right. \\
& -\frac{A \cos ^{2}(x)}{6720\left(1+\sigma_{1}\right)^{4}}\left(-6720 A^{2} \beta_{2} \sigma_{1}^{3} \alpha-20160 A^{2} \beta_{2} \sigma_{1}^{2} \alpha-6720 A^{2} \beta_{2} \alpha-20160 A^{2} \beta_{2} \sigma_{1} \alpha\right) \\
& -\frac{A}{6720\left(1+\sigma_{1}\right)^{4}}\left(6720 A^{2} \beta_{2} \sigma_{1} \alpha+1120 \Omega \beta_{1} \sigma_{1}^{2}+1120 \beta_{1} \sigma_{1}^{3}+1120 \beta_{1}\right. \\
& +3360 \beta_{1} \sigma_{1}^{2}+2240 \Omega \beta_{1} \sigma_{1}+3360 \beta_{1} \sigma_{1}+1120 \Omega \beta_{1} \\
& \left.\left.+6720 A^{2} \beta_{2} \sigma_{1}^{2} \alpha+2240 A^{2} \beta_{2} \sigma_{1}^{3} \alpha+2240 A^{2} \beta_{2} \alpha\right)\right] \sin (x) \\
& -\frac{A \cos ^{2}(x)}{6720\left(1+\sigma_{1}\right)^{4}}\left(-4480 \Omega A \beta_{2} \sigma_{1}-2240 \Omega A \beta_{2} \sigma_{1}^{2}-2240 \beta_{2} A-6720 A \beta_{2} \sigma_{1}^{2}\right. \\
& -13440 A \beta_{1} \alpha \sigma_{1}^{2}-2240 A \beta_{2} \sigma_{1}^{3}-4480 A \beta_{1} \alpha-2240 \Omega A \beta_{2} \\
& \left.-13440 A \beta_{1} \alpha \sigma_{1}-6720 A \beta_{2} \sigma_{1}-4480 A \beta_{1} \alpha \sigma_{1}^{3}\right) \\
& -\frac{A}{6720\left(1+\sigma_{1}\right)^{4}}\left(1120 \beta_{2} A+1120 \Omega A \beta_{2} \sigma_{1}^{2}+1120 A \beta_{2} \sigma_{1}^{3}+2240 A \beta_{1} \alpha\right. \\
& +2240 A \beta_{1} \alpha \sigma_{1}^{3}+1120 \Omega A \beta_{2}+3360 A \beta_{2} \sigma_{1}^{2}+2240 \Omega A \beta_{2} \sigma_{1} \\
& \left.+6720 A \beta_{1} \alpha \sigma_{1}+3360 A \beta_{2} \sigma_{1}+6720 A \beta_{1} \alpha \sigma_{1}^{2}\right) \\
& -\frac{A \cos (x)}{6720\left(1+\sigma_{1}\right)^{4}}\left(3360 \Omega A^{2} \delta+1120 \Omega A \beta_{2}-2240 \Omega \sigma_{2} \sigma_{1}-1120 \Omega \sigma_{2}\right. \\
& -1120 \Omega \sigma_{2} \sigma_{1}^{2}+1120 \Omega+2240 \Omega \sigma_{1}+2240 \Omega A \beta_{2} \sigma_{1} \\
& \left.+1120 \Omega \sigma_{1}^{2}+6720 \Omega A^{2} \delta \sigma_{1}+3360 \Omega A^{2} \delta \sigma_{1}^{2}+1120 \Omega A \beta_{2} \sigma_{1}^{2}\right) \\
& \left.-\frac{A \cos ^{3}(x)}{6720\left(1+\sigma_{1}\right)^{4}}\left(-6720 \Omega A^{2} \delta \sigma_{1}-3360 \Omega A^{2} \delta \sigma_{1}^{2}-3360 \Omega A^{2} \delta\right)\right\} t^{3} \\
& +\left\{-\frac{A \cos ^{3}(x)}{6720\left(1+\sigma_{1}\right)^{4}}\left(30240 A^{2} \delta \sigma_{1}^{2}+10080 A^{2} \delta \sigma_{1}^{3}+10080 A^{2} \delta+30240 A^{2} \delta \sigma_{1}\right)\right. \\
& -\frac{A}{6720\left(1+\sigma_{1}\right)^{4}}\left(-10080 A \beta_{2} \sigma_{1}^{2}-3360 \beta_{2} A-3360 A \beta_{2} \sigma_{1}^{3}-10080 A \beta_{2} \sigma_{1}\right) \\
& +\left[-\frac{A}{6720\left(1+\sigma_{1}\right)^{4}}\left(-10080 \beta_{1} \sigma_{1}^{2}-3360 \beta_{1}-3360 \beta_{1} \sigma_{1}^{3}-10080 \beta_{1} \sigma_{1}\right)\right. \\
& \left.-\frac{A \cos (x)}{6720\left(1+\sigma_{1}\right)^{4}}\left(-20160 A \gamma \sigma_{1}-6720 A \gamma \sigma_{1}^{3}-6720 A \gamma-20160 A \gamma \sigma_{1}^{2}\right)\right] \sin (x) \\
& -\frac{A \cos ^{2}(x)}{6720\left(1+\sigma_{1}\right)^{4}}\left(20160 A \beta_{2} \sigma_{1}+20160 A \beta_{2} \sigma_{1}^{2}+6720 A \beta_{2} \sigma_{1}^{3}+6720 \beta_{2} A\right)
\end{aligned}
$$




$$
\begin{gathered}
-\frac{A / \cos (x)}{6720\left(1+\sigma_{1}\right)^{4}}\left(-30240 A^{2} \delta \sigma_{1}^{2}-10080 A \beta_{2} \sigma_{1}+3360 \sigma_{2} \sigma_{1}^{3}-10080 A^{2} \delta\right. \\
+10080 \sigma_{2} \sigma_{1}-3360 A \beta_{2} \sigma_{1}^{3}-3360-30240 A^{2} \delta \sigma_{1}-3360 \beta_{2} A \\
+10080 \sigma_{2} \sigma_{1}^{2}+3360 \sigma_{2}-10080 \sigma_{1}^{2}-10080 A^{2} \delta \sigma_{1}^{3} \\
\left.\left.-10080 A \beta_{2} \sigma_{1}^{2}-3360 \sigma_{1}^{3}-10080 \sigma_{1}\right)\right\} t^{2} \\
-\frac{A \cos (x)}{6720\left(1+\sigma_{1}\right)^{4}}\left(6720+6720 \sigma_{1}^{4}+40320 \sigma_{1}^{2}+26880 \sigma_{1}^{3}+26880 \sigma_{1}\right) \\
-\frac{A}{6720\left(1+\sigma_{1}\right)^{4}}\left(-6720-26880 \sigma_{1}-26880 \sigma_{1}^{3}-6720 \sigma_{1}^{4}-40320 \sigma_{1}^{2}\right)
\end{gathered}
$$

$$
\begin{aligned}
& \theta_{2}=\left\{-\frac{A \cos (x)^{5}}{24\left(1+\sigma_{1}\right)^{2}}\left(-540 A^{4} \delta \alpha^{2}-540 A^{4} \delta \alpha^{2} \sigma_{1}+216 A^{4} b \delta \beta_{2}\right)\right. \\
& -\frac{A \cos (x)^{3}}{24\left(1+\sigma_{1}\right)^{2}}\left(-24 A^{3} b \beta_{2}^{2}+36 A^{3} \beta_{2} \alpha^{2}+24 A^{2} b \sigma_{2} \beta_{2}+36 A^{3} \beta_{2} \alpha^{2} \sigma_{1}+36 A^{2} \alpha^{2} \sigma_{1}\right. \\
& +36 A^{2} \alpha \gamma \sigma_{1}+702 A^{4} \delta \alpha^{2} \sigma_{1}-36 A^{2} \sigma_{2} \alpha^{2} \sigma_{1}-36 A^{2} \sigma_{2} \alpha^{2}+36 A^{2} \alpha^{2} \\
& \left.-24 A^{2} b \beta_{1} \gamma-288 A^{4} b \delta \beta_{2}-24 A^{2} b \beta_{2}+36 A^{2} \alpha \gamma+702 A^{4} \delta \alpha^{2}\right) \\
& +\left[-\frac{A \cos ^{2}(x)}{24\left(1+\sigma_{1}\right)^{2}}\left(-24 A^{2} b \gamma \sigma_{2}+36 A^{2} \beta_{1} \alpha^{2} \sigma_{1}-24 A^{2} b \beta_{1} \beta_{2}+36 A^{2} \beta_{1} \alpha^{2}+36 A^{2} \beta_{2} \alpha\right.\right. \\
& \left.+24 A^{3} b \gamma \beta_{2}+24 A^{2} b \gamma+180 A^{4} b \gamma \delta+36 A^{2} \beta_{2} \sigma_{1} \alpha\right)+\frac{9 A^{5} b \gamma \delta \cos ^{4}(x)}{\left(1+\sigma_{1}\right)^{2}} \\
& -\frac{A \cos (x)}{24\left(1+\sigma_{1}\right)^{2}}\left(-6 \alpha A \sigma_{1}+6 b A \beta_{1}+18 A^{3} b \beta_{1} \delta-72 \alpha A^{3} \delta-96 A^{3} \gamma \alpha^{2} \sigma_{1}+48 A^{3} b \gamma \beta_{2}\right. \\
& -6 A^{2} \beta_{2} \sigma_{1} \alpha-6 A^{2} \beta_{2} \alpha-96 A^{3} \gamma \alpha^{2}+6 \alpha A \sigma_{2}-6 \alpha A-6 A b \beta_{1} \sigma_{2} \\
& \left.-72 \alpha A^{3} \delta \sigma_{1}+6 \alpha A \sigma_{2} \sigma_{1}+6 A^{2} b \beta_{1} \beta_{2}\right) \\
& -\frac{A \cos ^{3}(x)}{24\left(1+\sigma_{1}\right)^{2}}\left(192 A^{3} \gamma \alpha^{2} \sigma_{1}+192 A^{3} \gamma \alpha^{2}+108 \alpha A^{3} \delta-96 A^{3} b \gamma \beta_{2}\right. \\
& \left.+108 \alpha A^{3} \delta \sigma_{1}-54 A^{3} b \beta_{1} \delta\right) \\
& -\frac{A}{24\left(1+\sigma_{1}\right)^{2}}\left(-12 A^{2} b \gamma-12 A^{2} \beta_{2} \alpha-12 A^{3} b \gamma \beta_{2}-36 A^{4} b \gamma \delta-6 A^{2} \beta_{1} \alpha^{2}-12 A^{2} \beta_{2} \sigma_{1} \alpha\right. \\
& \left.\left.+12 A^{2} b \gamma \sigma_{2}-6 A^{2} \beta_{1} \alpha^{2} \sigma_{1}\right)\right] \sin (x)
\end{aligned}
$$




$$
\begin{aligned}
& -\frac{A \cos ^{2}(x)}{24\left(1+\sigma_{1}\right)^{2}}\left(3 A b+72 A^{4} b \delta \beta_{2}+48 A^{3} b \gamma^{2}+3 A b \sigma_{2}^{2}+189 A^{5} b \delta^{2}+6 A \beta_{1} \alpha \sigma_{1}\right. \\
& -6 A^{2} b \sigma_{2} \beta_{2}+6 A \beta_{1} \alpha-3 A b \beta_{1}^{2}+192 A^{3} \beta_{2} \alpha^{2}+192 A^{3} \beta_{2} \alpha^{2} \sigma_{1} \\
& \left.+72 A^{3} b \delta+6 A^{2} b \beta_{2}-45 A^{3} b \beta_{2}^{2}-72 A^{3} b \delta \sigma_{2}-6 A b \sigma_{2}\right) \\
& -\frac{A \cos (x)}{24\left(1+\sigma_{1}\right)^{2}}\left(72 A^{4} b \delta \beta_{2}-198 A^{4} \delta \alpha^{2} \sigma_{1}-30 A^{3} \beta_{2} \alpha^{2}-30 A^{2} \alpha^{2} \sigma_{1}+24 A^{3} b \beta_{2}^{2}\right. \\
& -30 A^{3} \beta_{2} \alpha^{2} \sigma_{1}+30 A^{2} \sigma_{2} \alpha^{2}-24 A^{2} b \sigma_{2} \beta_{2}+12 A^{2} b \beta_{1} \gamma+30 A^{2} \sigma_{2} \alpha^{2} \sigma_{1} \\
& \left.-198 A^{4} \delta \alpha^{2}-30 A^{2} \alpha \gamma \sigma_{1}+24 A^{2} b \beta_{2}-30 A^{2} \alpha \gamma-30 A^{2} \alpha^{2}\right) \\
& -\frac{A \cos ^{4}(x)}{24\left(1+\sigma_{1}\right)^{2}}\left(-405 A^{5} b \delta^{2}-192 A^{3} \beta_{2} \alpha^{2}+54 A^{3} b \delta \sigma_{2}-54 A^{4} b \delta \beta_{2}-48 A^{3} b \gamma^{2}\right. \\
& \left.+48 A^{3} b \beta_{2}^{2}-54 A^{3} b \delta-192 A^{3} \beta_{2} \alpha^{2} \sigma_{1}\right)-\frac{81 A^{6} b \delta^{2} \cos ^{6}(x)}{8\left(1+\sigma_{1}\right)^{2}} \\
& -\frac{A}{24\left(1+\sigma_{1}\right)^{2}}\left(-12 A^{3} b \gamma^{2}-18 A^{4} b \delta \beta_{2}-3 A b-3 A b \sigma_{2}^{2}-3 A^{3} b \beta_{2}^{2}\right. \\
& +6 A^{2} b \sigma_{2} \beta_{2}-3 A \beta_{1} \alpha-27 A^{5} b \delta^{2}-3 A \beta_{1} \alpha \sigma_{1}-24 A^{3} \beta_{2} \alpha^{2} \sigma_{1}-18 A^{3} b \delta \\
& \left.\left.-6 A^{2} b \beta_{2}+18 A^{3} b \delta \sigma_{2}-24 A^{3} \beta_{2} \alpha^{2}+6 A b \sigma_{2}\right)\right\} t^{4} \\
& +\left\{-\frac{A\left(-48 A^{2} \alpha \gamma-48 A^{2} \alpha \gamma \sigma_{1}\right) \cos ^{3}(x)}{24\left(1+\sigma_{1}\right)^{2}}-\frac{A\left(40 A^{2} \alpha \gamma+40 A^{2} \alpha \gamma \sigma_{1}\right) \cos (x)}{24\left(1+\sigma_{1}\right)^{2}}\right. \\
& -\frac{A\left(4 A \beta_{1} \alpha+4 A \beta_{1} \alpha \sigma_{1}\right)}{24\left(1+\sigma_{1}\right)^{2}}-\frac{A\left(-8 A \beta_{1} \alpha-8 A \beta_{1} \alpha \sigma_{1}\right) \cos ^{2}(x)}{24\left(1+\sigma_{1}\right)^{2}} \\
& +\left[-\frac{A\left(-48 A^{2} \beta_{2} \sigma_{1} \alpha-48 A^{2} \beta_{2} \alpha\right)}{24\left(1+\sigma_{1}\right)^{2}} \cos ^{2}(x)-\frac{A\left(-144 \alpha A^{3} \delta \sigma_{1}-144 \alpha A^{3} \delta\right) \cos ^{3}(x)}{24 /\left(1+\sigma_{1}\right)^{2}}\right. \\
& -\frac{A \cos (x)}{24\left(1+\sigma_{1}\right)^{2}}\left(8 \alpha A+96 \alpha A^{3} \delta \sigma_{1}-8 \alpha A \sigma_{2} \sigma_{1}-8 \alpha A \sigma_{2}+8 A^{2} \beta_{2} \alpha+96 \alpha A^{3} \delta\right. \\
& \left.+8 A^{2} \beta_{2} \sigma_{1} \alpha+8 \alpha A \sigma_{1}\right) \\
& \left.\left.-\frac{A}{24\left(1+\sigma_{1}\right)^{2}}\left(16 A^{2} \beta_{2} \alpha+16 A^{2} \beta_{2} \sigma_{1} \alpha\right)\right] \sin (x)\right\} t^{3} \\
& +\left\{-\frac{A \cos (x)}{24\left(1+\sigma_{1}\right)^{2}}\left(12-48 A^{2} b \beta_{2}+120 A^{2} \alpha^{2} \sigma_{1}^{2}-48 A^{2} \sigma_{1} b \beta_{2}+24 \sigma_{1}+12 \sigma_{1}^{2}\right.\right. \\
& \left.-12 \sigma_{1} a \beta_{1}+240 A^{2} \alpha^{2} \sigma_{1}-12 a \beta_{1}+120 A^{2} \alpha^{2}\right) \\
& -\frac{A \cos ^{3}(x)}{24\left(1+\sigma_{1}\right)^{2}}\left(48 A^{2} b \beta_{2}-144 A^{2} \alpha^{2} \sigma_{1}^{2}-288 A^{2} \alpha^{2} \sigma_{1}-144 A^{2} \alpha^{2}+48 A^{2} \sigma_{1} b \beta_{2}\right)
\end{aligned}
$$




$$
\begin{aligned}
& -\frac{A}{24\left(1+\sigma_{1}\right)^{2}}\left(24 A a \gamma+36 A^{3} b \delta+12 A^{2} b \beta_{2}-12 A b \sigma_{2}+12 A b+36 A^{3} \sigma_{1} b \delta\right. \\
& \left.+24 A \sigma_{1} a \gamma+12 A \sigma_{1} b-12 A \sigma_{1} b \sigma_{2}+12 A^{2} \sigma_{1} b \beta_{2}\right) \\
& +\left[-\frac{A \cos ^{2}(x)}{24\left(1+\sigma_{1}\right)^{2}}\left(-48 A^{2} b \gamma-108 a \delta A^{2}-108 \sigma_{1} a \delta A^{2}-48 A^{2} \sigma_{1} b \gamma\right)\right. \\
& -\frac{A}{24\left(1+\sigma_{1}\right)^{2}}\left(12 \sigma_{1} a \beta_{2} A+36 \sigma_{1} a \delta A^{2}-12 a \sigma_{2}+12 a \beta_{2} A-12 \sigma_{1} a \sigma_{2}\right. \\
& \left.+12 \sigma_{1} a+24 A^{2} b \gamma+36 a \delta A^{2}+12 a+24 A^{2} \sigma_{1} b \gamma\right) \\
& -\frac{A \cos (x)}{24\left(1+\sigma_{1}\right)^{2}}\left(240 \alpha A \sigma_{1}+120 \alpha A-48 a \beta_{2} A-12 b A \beta_{1}-48 \sigma_{1} a \beta_{2} A\right. \\
& \left.\left.+120 \alpha A \sigma_{1}^{2}-12 \sigma_{1} b A \beta_{1}\right)\right] \sin (x) \\
& -\frac{A \cos ^{2}(x)}{24\left(1+\sigma_{1}\right)^{2}}\left(-12 A b+12 A b \sigma_{2}-144 A^{3} \sigma_{1} b \delta-144 A^{3} b \delta-12 A^{2} b \beta_{2}-48 A a r\right. \\
& \left.-12 A^{2} \sigma_{1} b \beta_{2}-48 A \sigma_{1} a \gamma-12 A \sigma_{1} b+12 A \sigma_{1} b \sigma_{2}\right) \\
& \left.-\frac{A\left(108 A^{3} b \delta+108 A^{3} \sigma_{1} b \delta\right) \cos ^{4}(x)}{24\left(1+\sigma_{1}\right)^{2}}\right\} t^{2} \\
& \times\left\{\frac{A\left(-48 \alpha A-96 \alpha A \sigma_{1}-48 \alpha A \sigma_{1}^{2}\right) \cos (x) \sin (x)}{24\left(1+\sigma_{1}\right)^{2}}+-\frac{A\left(-24-48 \sigma_{1}-24 \sigma_{1}^{2}\right) \cos (x)}{24\left(1+\sigma_{1}\right)^{2}}\right\} t \\
& -\frac{A\left(24+48 \sigma_{1}+24 \sigma_{1}^{2}\right) \cos (x)}{24\left(1+\sigma_{1}\right)^{2}}-\frac{A\left(-24-48 \sigma_{1}-24 \sigma_{1}^{2}\right)}{24\left(1+\sigma_{1}\right)^{2}} .
\end{aligned}
$$

Now we make calculations for the results obtained by the variational iteration method using the Maple software package with the following arbitrary constants:

$$
a=0.5, \quad A=0.001, \quad b=0.5, \quad \alpha=1, \quad \beta_{1}=\beta_{2}=0.05, \quad \gamma=1, \quad \delta=0.8
$$

\subsection{Special Case}

(1) If we take into our consideration the first iteration (i.e., $u=u_{1}$ and $\theta=\theta_{1}$ ). See Figures 15, 16, 17, and 18.

(2) If the magnetic field and rotation are neglected, the components of the displacement $u_{2}$ and temperature $\theta_{2}$ take the following forms. See Figures 19 and 20. 


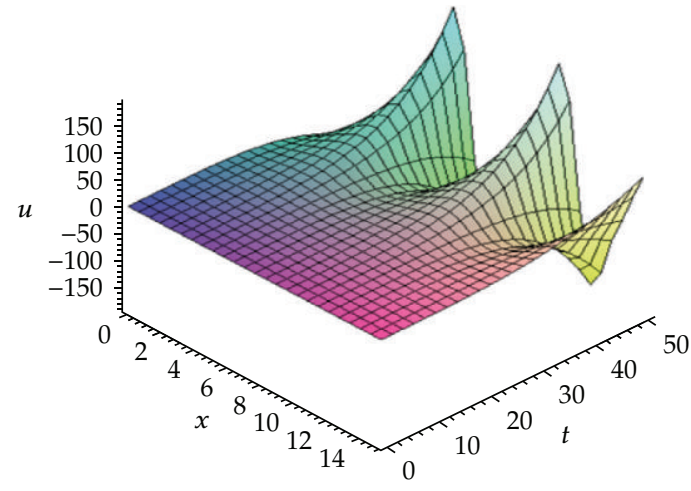

(a)

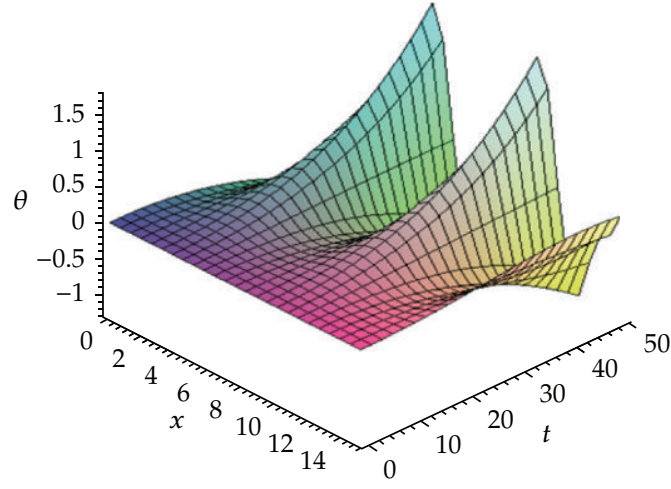

(b)

Figure 11: Variations of the displacement $u_{2}$ and temperature $\theta_{2}$ for various values of the axis $x$ and time $t$ when $\Omega=0.1, \sigma_{1}=0.2, \sigma_{2}=0.1$.

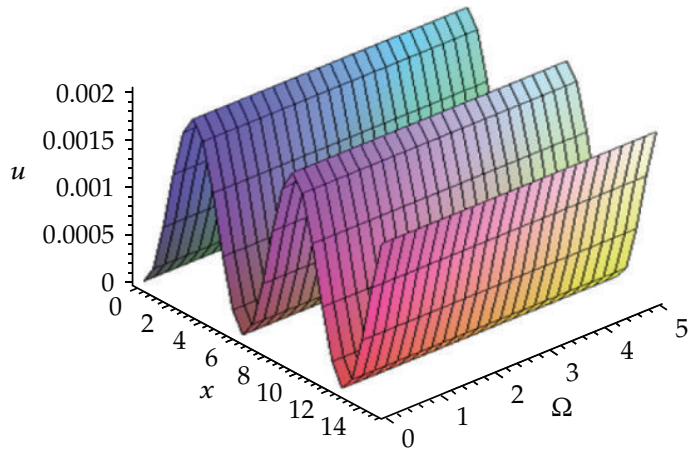

(a)

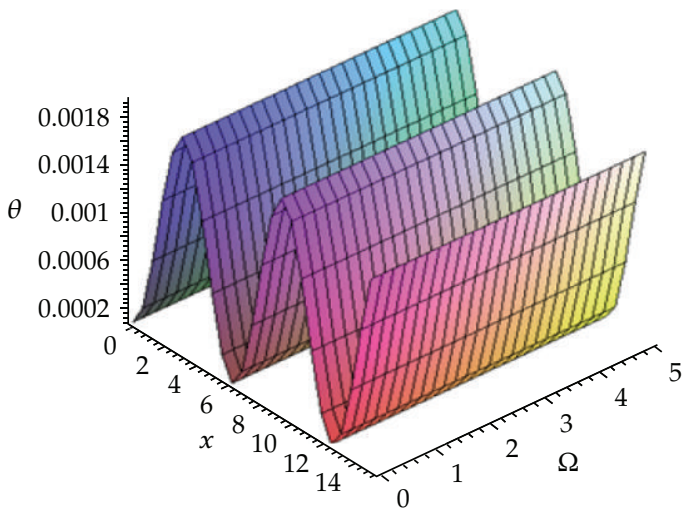

(b)

Figure 12: Variations of the displacement $u_{2}$ and temperature $\theta_{2}$ for various values of the axis $x$ and rotation $\Omega$ when $t=0.1, \sigma_{1}=0.2, \sigma_{2}=0.1$.

\section{Discussion}

With the view of illustrating the theoretical results obtained in the preceding sections, a numerical result is calculated for the homotopy perturbation method and variational iteration method.

Figures (1-10) illustrate the influences of time $t$, rotation $\Omega$, and sensitive pats of the magnetic field $\sigma_{1}$ and $\sigma_{2}$ for the iterations $\left(u=V_{0}+V_{1}+V_{2}\right.$ and $\left.\theta=\Theta_{0}+\Theta_{1}+\Theta_{2}\right)$ and ( $u=V_{0}+V_{1}$ and $\left.\theta=\Theta_{0}+\Theta_{1}\right)$, and if the rotation and magnetic field neglected, respectively, respect to the coordinate $x$ for the homotopy perturbation method. Figures (11-20) illustrate the influences of time $t$, rotation $\Omega$, and sensitive pats of the magnetic field $\sigma_{1}$ and $\sigma_{2}$ for the iterations $\left(u=V_{2}, \theta=\theta_{2}\right.$ and $u=V_{1}$ and $\left.\theta=\theta_{1}\right)$, and if the rotation and magnetic field have been neglected, respectively, respect to the coordinate $x$ for the variational iteration method.

From Figures 1 and 11, it is concluded that the displacement $u$ and temperature $\theta$ start from their maximum values, decrease and increase periodically with an increasing of 


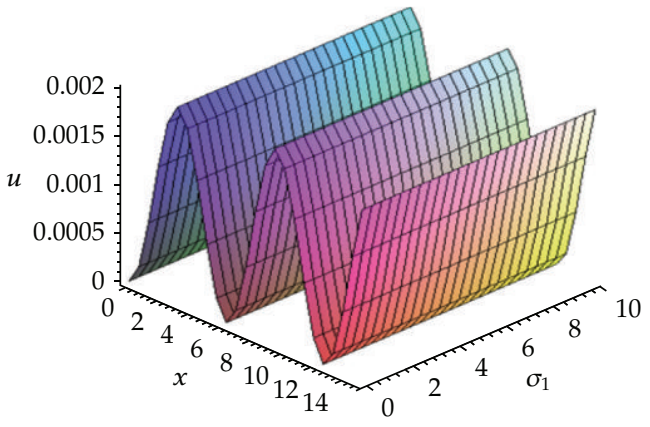

(a)

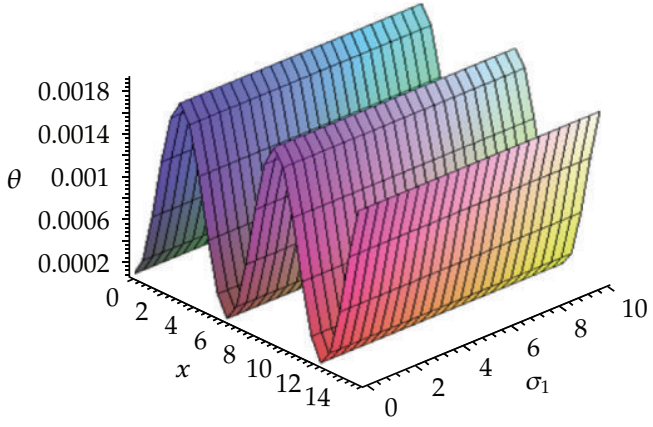

(b)

Figure 13: Variations of the displacement $u_{2}$ and temperature $\theta_{2}$ for various values of the axis $x$ and magnetic field $\sigma_{1}$ when $t=0.1, \Omega=0.1, \sigma_{2}=0.1$.

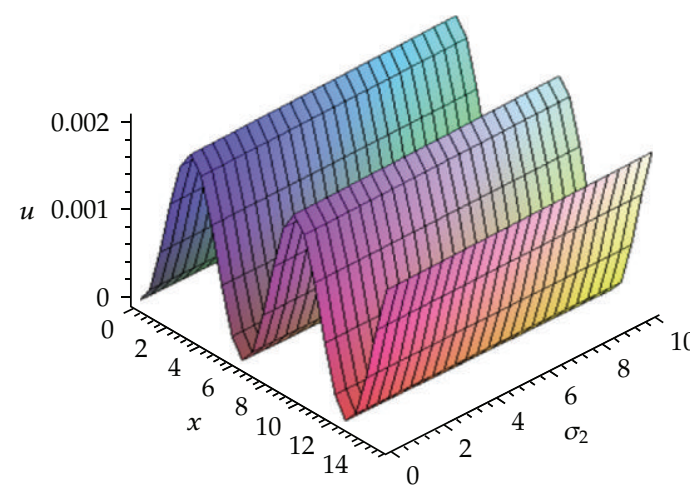

(a)

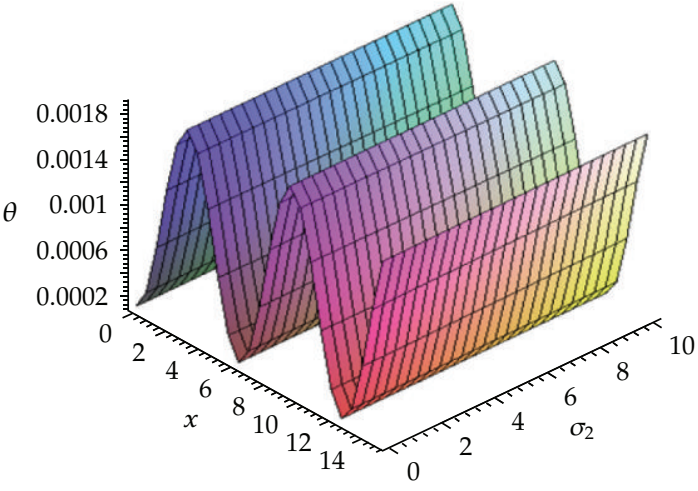

(b)

Figure 14: Variations of the displacement $u_{2}$ and temperature $\theta_{2}$ for various values of the axis $x$ and magnetic field $\sigma_{2}$ when $t=0.1, \Omega=0.1, \sigma_{1}=0.1$.

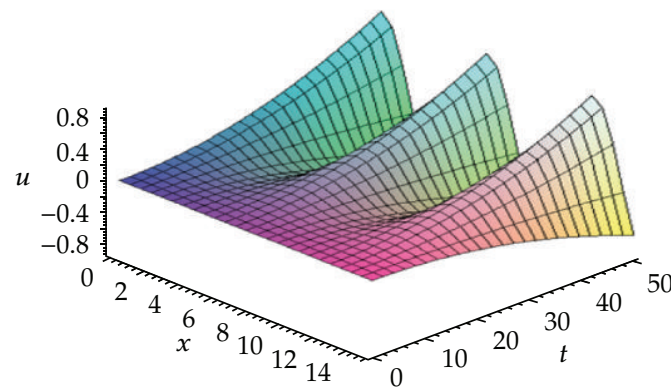

(a)

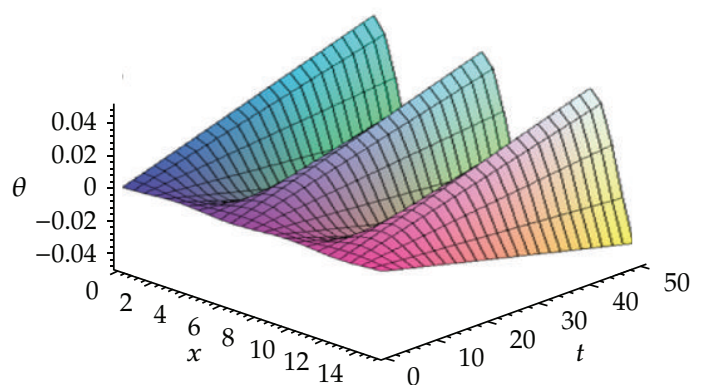

(b)

Figure 15: Variations of the displacement $u_{1}$ and temperature $\theta_{1}$ for various values of the axis $x$ and time $t$ when $\Omega=0.1, \sigma_{1}=0.2, \sigma_{2}=0.1$. 


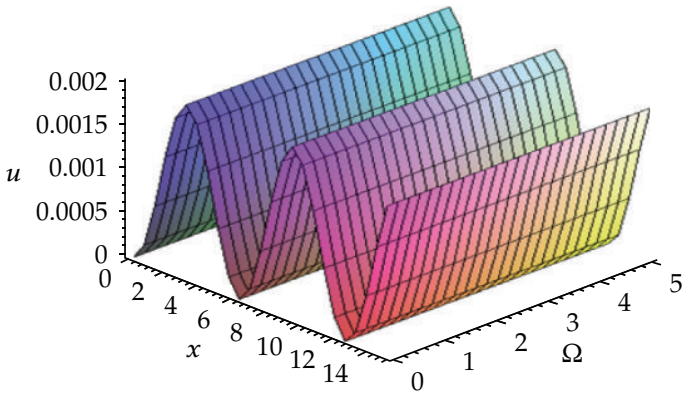

(a)

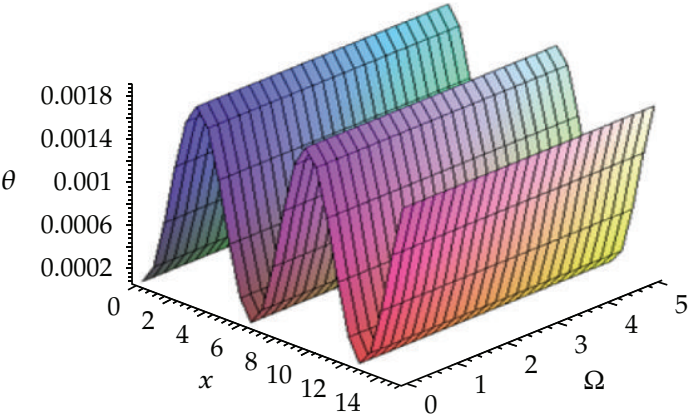

(b)

Figure 16: Variations of the displacement $u_{1}$ and temperature $\theta_{1}$ for various values of the axis $x$ and rotation $\Omega$ when $t=0.1, \sigma_{1}=0.2, \sigma_{2}=0.1$.

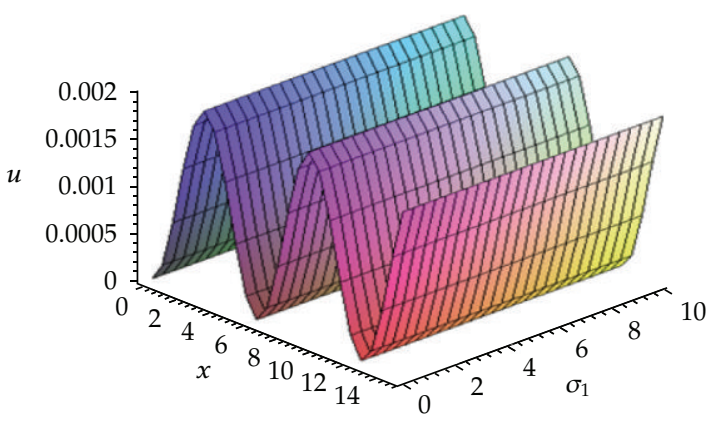

(a)

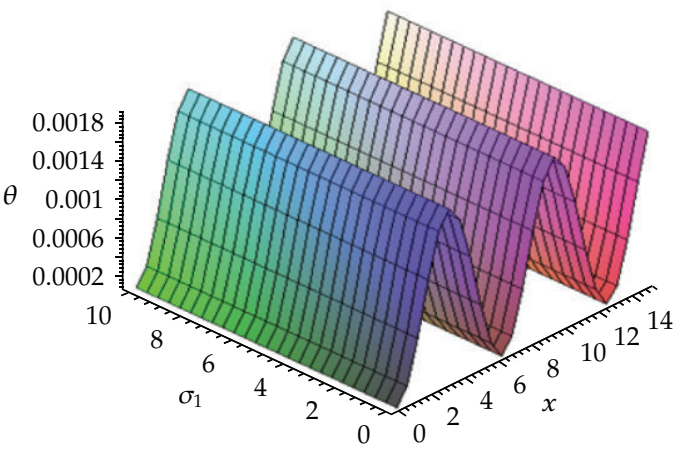

(b)

Figure 17: Variations of the displacement $u_{1}$ and temperature $\theta_{1}$ for various values of the axis $x$ and magnetic field $\sigma_{1}$ when $t=0.1, \Omega=0.1, \sigma_{2}=0.1$.

the coordinate $x$, also, it is obvious that their values take the minimum values and increases with the increasing values of the time $t$. From Figures $2,3,4,12,13,14$, it is seen that the components of the displacement $u$ and temperature $\theta$ begin from the minimum values near zero increase and then decrease periodically with the coordinate $x$, it is clear also that there are a sligh increasing with an increasing of the sensitive parts of the magnetic field, also, one can see that $u$ and $\theta$ decrease with an increasing of the rotation $\Omega$.

Figures 5-8 and 15-18 display the first iteration with respect to the homotopy perturbation method and variational iteration method on the influences of the parameters time $t$, rotation $\Omega$, and sensitive pats of the magnetic field $\sigma_{1}$ and $\sigma_{2}$ to obtain the displacement and the temperature components on the medium due to the harmonic wave propagation. It is shown that the increasing of the coordinate $x$ sensitive an increasing and dereasing on them periodically due to appearance of the pairs $(\cos , \sin )$ in the initial condition and the approximate solutions; it is also clear that the components begin from their minimum values and increase absolutely with the variation of the time $t$. With the variations of the rotation and magnetic field tends to slightly affect on the displacment and the temperature. 


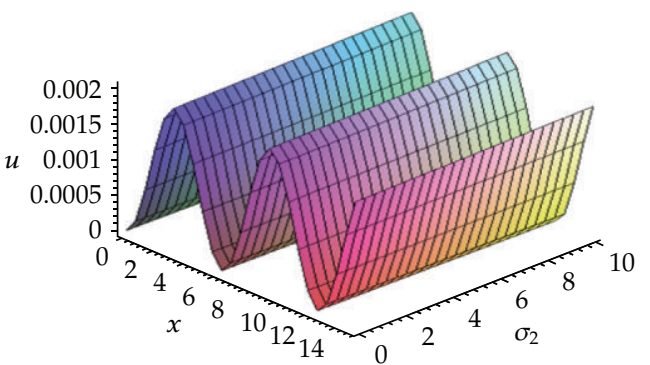

(a)

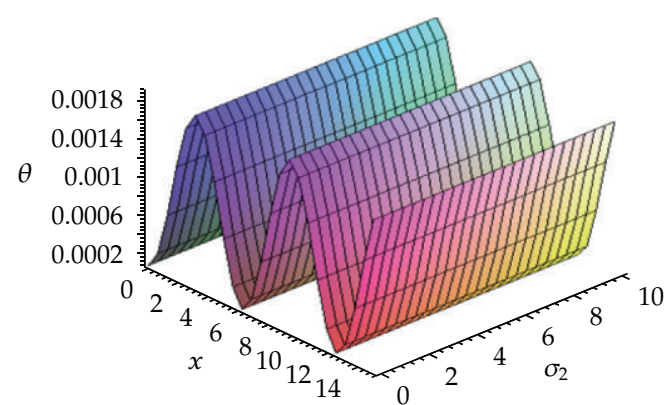

(b)

Figure 18: Variations of the displacement $u_{1}$ and temperature $\theta_{1}$ for various values of the axis $x$ and magnetic field $\sigma_{2}$ when $t=0.1, \Omega=0.1, \sigma_{1}=0.1$.

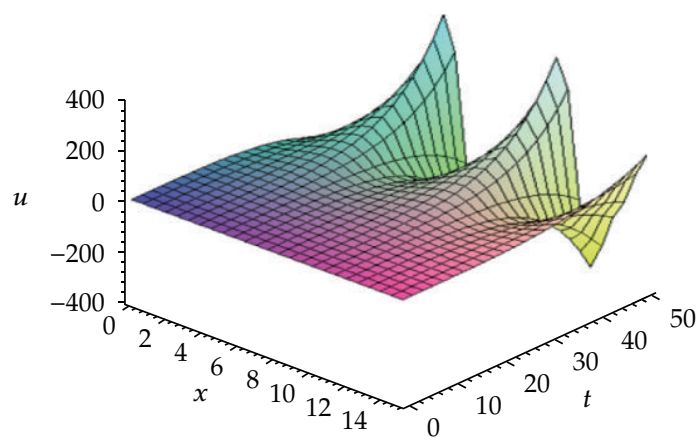

(a)

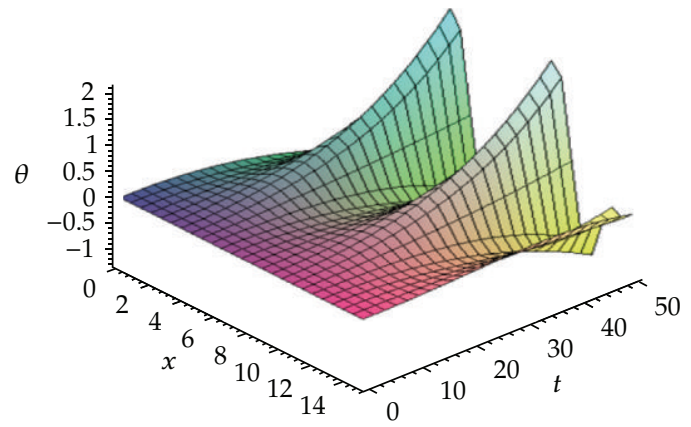

(b)

Figure 19: Variations of the displacement $u_{2}$ and temperature $\theta_{2}$ for various values of the axis $x$ and time $t$ $\left(u=u_{2}\right.$ and $\left.\theta=\theta_{2}\right)$ when $\Omega=\sigma_{1}=\sigma_{2}=0$.

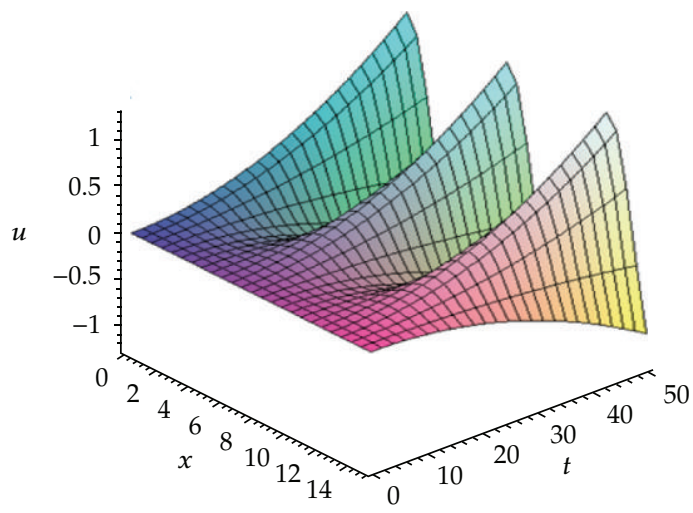

(a)

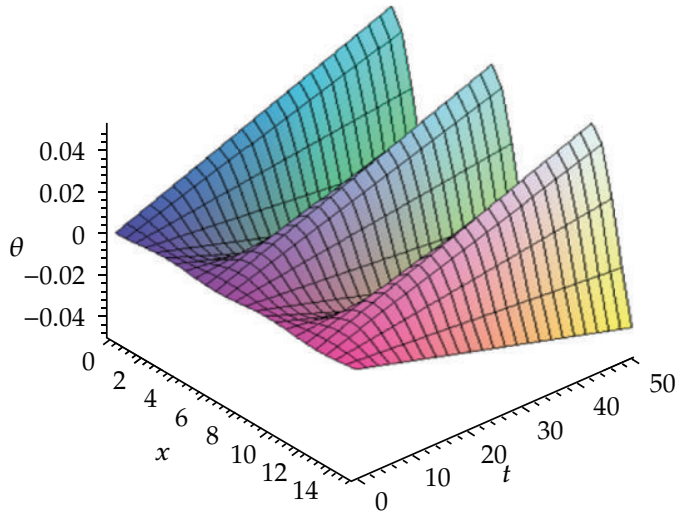

(b)

Figure 20: Variations of the displacement $u_{1}$ and temperature $\theta_{1}$ for various values of the axis $x$ and time $t$ $\left(u=u_{1}\right.$ and $\left.\theta=\theta_{1}\right)$ when $\Omega=\sigma_{1}=\sigma_{2}=0$. 
It seems too that there are a clear differs between the results obtained by the HPM and the corresponding results obtained by VIM resultant to the appearance of the high order of time in VIM tends to the high values of the approximate solution comparing with the results obtained by HPM. Because of the results obtained, we concluded that the homotopy perturbation method is more effective and powerful than the variational iteration method.

On the other hand, from Figures 9, 10, 19, and 20, it is obvious that if the rotaion and magnetic field are neglected, the approximate solutions by HPM and VIM in first iteration are the same in both methods and agree with the results obtained by Sweilam and Khader [1].

\section{References}

[1] N. H. Sweilam and M. M. Khader, "Variational iteration method for one dimensional nonlinear thermoelasticity," Chaos, Solitons and Fractals, vol. 32, no. 1, pp. 145-149, 2007.

[2] N. H. Sweilam, "Harmonic wave generation in non linear thermoelasticity by variational iteration method and Adomian's method," Journal of Computational and Applied Mathematics, vol. 207, no. 1, pp. 64-72, 2007.

[3] H. N. Sweilam, M. M. Khader, and F. R. Al-Bar, "On the numerical simulation of population dynamics with density-dependent migrations and the Allee effects," Journal of Physics: Conference Series, vol. 96, no. 1, Article ID 012008, 2008.

[4] N. H. Sweilam, M. M. Khader, and R. F. Al-Bar, "Nonlinear focusing Manakov systems by variational iteration method and adomian decomposition method," Journal of Physics: Conference Series, vol. 96, no. 1, Article ID 012164, 2008.

[5] A. N. Abd-Alla, A. F. Ghaleb, and G. A. Maugin, "Harmonic wave generation in nonlinear thermoelasticity," International Journal of Engineering Science, vol. 32, no. 7, pp. 1103-1116, 1994.

[6] C. A. De Moura, "A linear uncoupling numerical scheme for the nonlinear coupled thermodynamics equations," in Lecture Notes in Mathematics, V. Pereyra and A. Reinoze, Eds., vol. 1005, pp. 204-211, Springer, Berlin, Germany, 1983.

[7] M. Slemrod, "Global existence, uniqueness, and asymptotic stability of classical smooth solutions in one-dimensional nonlinear thermoelasticity," Archive for Rational Mechanics and Analysis, vol. 76, no. 2, pp. 97-133, 1981.

[8] J.H. He, "Some asymptotic methods for strongly nonlinear equations," International Journal of Modern Physics B, vol. 20, no. 10, pp. 1141-1199, 2006.

[9] J. H. He, Non-Perturbative Methods for Strongly Nonlinear Problems, Dissertation.de Verlag im Internet $\mathrm{GmbH}$, Berlin, Germany, 2006.

[10] J. H. He and X. H. Wu, "Construction of solitary solution and compacton-like solution by variational iteration method," Chaos, Solitons and Fractals, vol. 29, no. 1, pp. 108-113, 2006.

[11] J. H. He, Y. Q. Wan, and Q. Guo, "An iteration formulation for normalized diode characteristics," International Journal of Circuit Theory and Applications, vol. 32, no. 6, pp. 629-632, 2004.

[12] J. H. He, "A simple perturbation approach to Blasius equation," Applied Mathematics and Computation, vol. 140, no. 2-3, pp. 217-222, 2003.

[13] J. H. He, "Variational iteration method for autonomous ordinary differential systems," Applied Mathematics and Computation, vol. 114, no. 2-3, pp. 115-123, 2000.

[14] J. H. He, "Variational iteration method-a kind of non-linear analytical technique: some examples," International Journal of Non-Linear Mechanics, vol. 34, no. 4, pp. 699-708, 1999.

[15] J. H. He, "Approximate analytical solution for seepage flow with fractional derivatives in porous media," Computer Methods in Applied Mechanics and Engineering, vol. 167, no. 1-2, pp. 57-68, 1998.

[16] A. M. Wazwaz, "The variational iteration method for solving nonlinear singular boundary value problems arising in various physical models," Communications in Nonlinear Science and Numerical Simulation, vol. 16, no. 10, pp. 3881-3886, 2011.

[17] M. A. Noor, K. I. Noor, and S. T. Din, "Variational iteration method for solving sixth-order boundary value problems," Communications in Nonlinear Science and Numerical Simulation, vol. 14, no. 6, pp. 2571-2580, 2009. 
[18] F. Soltanian, S. M. Karbassi, and M. M. Hosseini, “Application of He's variational iteration method for solution of differential-algebraic equations," Chaos, Solitons and Fractals, vol. 41, no. 1, pp. 436-445, 2009.

[19] S. M. Hassan and N. M. Alotaibi, "Solitary wave solutions of the improved KdV equation by VIM," Applied Mathematics and Computation, vol. 217, no. 6, pp. 2397-2403, 2010.

[20] E. Yusufoğlu, "Two convergence theorems of variational iteration method for ordinary differential equations," Applied Mathematics Letters. In press.

[21] J. H. He, "Approximate solution of nonlinear differential equations with convolution product nonlinearities," Computer Methods in Applied Mechanics and Engineering, vol. 167, no. 1-2, pp. 69-73, 1998.

[22] J. H. He, "Homotopy perturbation technique," Computer Methods in Applied Mechanics and Engineering, vol. 178, no. 3-4, pp. 257-262, 1999.

[23] A. Yildirim, "Applying he's variational iteration method for solving differential-difference equation," Mathematical Problems in Engineering, vol. 2008, Article ID 869614, 7 pages, 2008.

[24] M. A. Noor and S. T. Mohyud-Din, "Variational iteration method for solving higher-order nonlinear boundary value problems using He's polynomials," International Journal of Nonlinear Sciences and Numerical Simulation, vol. 9, no. 2, pp. 141-156, 2008.

[25] S. T. Mohyud-Din, M. A. Noor, and K. I. Noor, "Solving second-order singular problems using He's polynomials," World Applied Sciences Journal, vol. 6, no. 6, pp. 769-775, 2009.

[26] S. T. Mohyud-Din, M. A. Noor, and K. I. Noor, "Traveling wave solutions of seventh-order generalized $\mathrm{KdV}$ equations using he's polynomials," International Journal of Nonlinear Sciences and Numerical Simulation, vol. 10, no. 2, pp. 227-233, 2009.

[27] S. T. Mohyud-Din and A. Yildirim, "Solving nonlinear boundary value problems using He's polynomials and Padé approximants," Mathematical Problems in Engineering, vol. 2009, Article ID 690547, 17 pages, 2009.

[28] S. T. Mohyud-Din, A. Yildirim, S. Anl Sezer, and M. Usman, "Modified variational iteration method for free-convective boundary-layer equation using padé approximation," Mathematical Problems in Engineering, vol. 2010, Article ID 318298, 11 pages, 2010.

[29] S. T. Mohyud-Din and M. A. Noor, "Homotopy perturbation method for solving fourth-order boundary value problems," Mathematical Problems in Engineering, Article ID 98602, 15 pages, 2007.

[30] S. T. Mohyud-Din and M. A. Noor, "Homotopy perturbation method for solving partial differential equations," Zeitschrift fur Naturforschung, vol. 64, no. 3-4, pp. 157-170, 2009.

[31] S. T. Mohyud-Din, M. A. Noor, and K. I. Noor, "Some relatively new techniques for nonlinear problems," Mathematical Problems in Engineering, vol. 2009, Article ID 234849, 25 pages, 2009. 


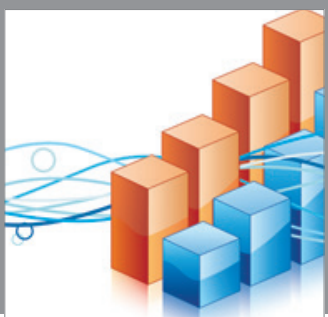

Advances in

Operations Research

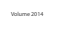

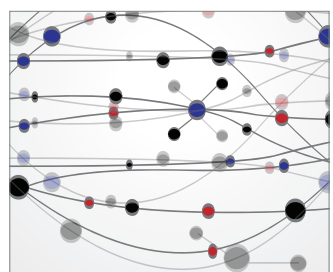

\section{The Scientific} World Journal
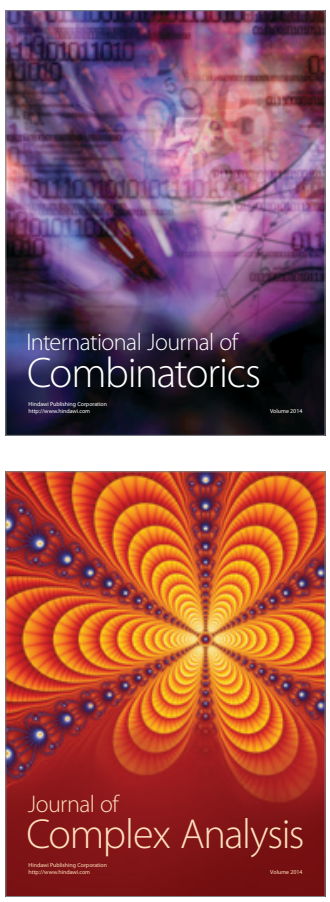

International Journal of

Mathematics and

Mathematical

Sciences
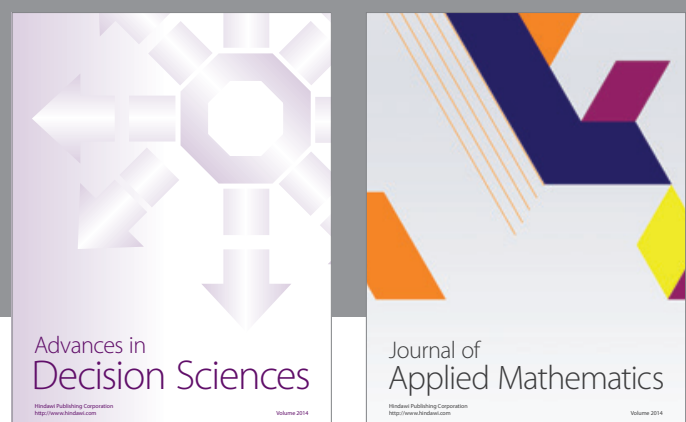

Journal of

Applied Mathematics
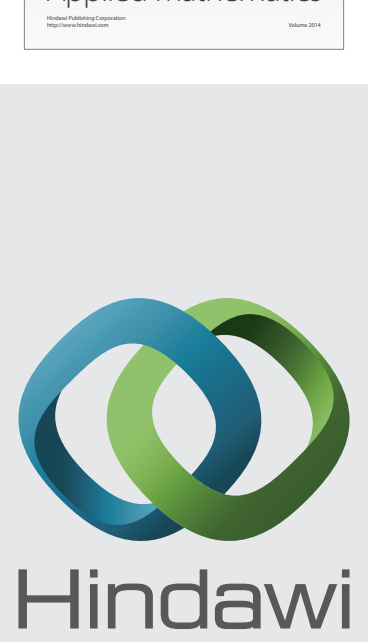

Submit your manuscripts at http://www.hindawi.com
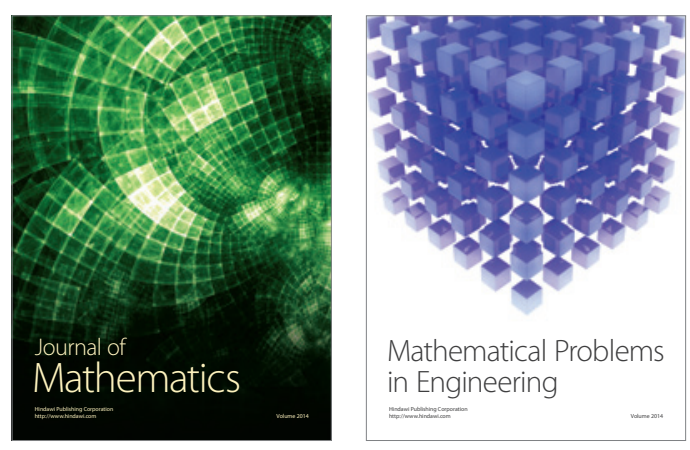

Mathematical Problems in Engineering
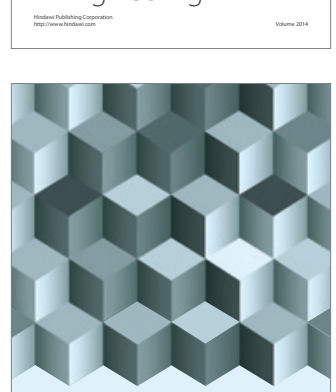

Journal of

Function Spaces
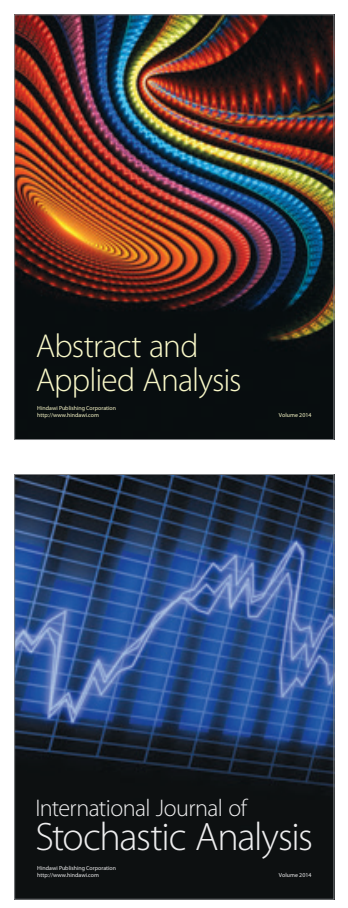

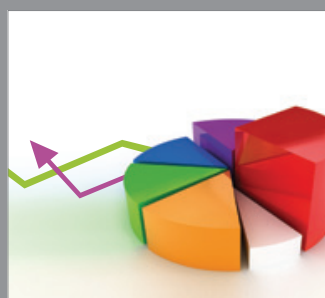

ournal of

Probability and Statistics

Promensencen
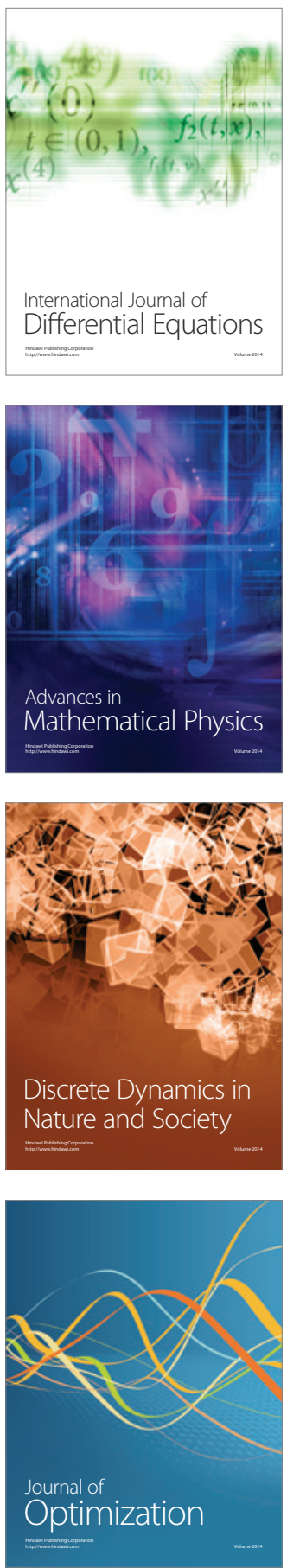